\title{
Kinetic Mixing of the Photon with Hidden $U(1) s$ in String Phenomenology
}

\author{
S. A. Abel ${ }^{a}$, M. D. Goodsell ${ }^{b}$, J. Jaeckel ${ }^{a, c}$, V. V. Khoze ${ }^{a}$, A. Ringwald ${ }^{d}$ \\ ${ }^{a}$ Institute for Particle Physics Phenomenology, Durham University, Durham, DH1 3LE, \\ United Kingdom \\ ${ }^{b}$ Laboratoire de Physique Théorique et Hautes Energies, Tour 24-25, 5eme tage, Boite \\ 126, 4 place Jussieu, F-75252 Paris Cedex 05, France \\ ${ }^{c}$ Institut für Theoretische Physik, Universität Heidelberg, Philosophenweg 16, D-69120 \\ Heidelberg, Germany \\ ${ }^{d}$ Deutsches Elektronen-Synchrotron DESY, Notkestraße 85, D-22607 Hamburg, Germany \\ E-mail: s.a.abel@durham.ac.uk, goodsell@lpthe.jussieu.fr, \\ joerg.jaeckel@durham.ac.uk, valya.khoze@durham.ac.uk, \\ andreas.ringwald@desy.de
}

ABSTRACT: Embeddings of the standard model in type II string theory typically contain a variety of $U(1)$ gauge factors arising from D-branes in the bulk. In general, there is no reason why only one of these - the one corresponding to weak hypercharge - should be massless. Observations require that standard model particles must be neutral (or have an extremely small charge) under additional massless $U(1)$ s, i.e. the latter have to belong to a so called hidden sector. The exchange of heavy messengers, however, can lead to a kinetic mixing between the hypercharge and the hidden-sector $U(1) \mathrm{s}$, that is testable with near future experiments. This provides a powerful probe of the hidden sectors and, as a consequence, of the string theory realisation itself. In the present paper, we show, using a variety of methods, how the kinetic mixing can be derived from the underlying type II string compactification, involving supersymmetric and nonsupersymmetric configurations of Dbranes, both in large volumes and in warped backgrounds with fluxes. We first demonstrate by explicit example that kinetic mixing occurs in a completely supersymmetric set-up where we can use conformal field theory techniques. We then develop a supergravity approach which allows us to examine the phenomenon in more general backgrounds, where we find that kinetic mixing is natural in the context of flux compactifications. We discuss the phenomenological consequences for experiments at the low-energy frontier, searching for signatures of light, sub-electronvolt or even massless hidden-sector $U(1)$ gauge bosons and minicharged particles. 


\section{Contents}

1. Introduction 1

1.1 Review: detection of hidden-sector $U(1)$ s and current limits 3

1.2 Overview: kinetic versus mass mixing in type II string theory 6

2. The CFT computation of kinetic mixing: generalities 8

2.1 When can we have kinetic mixing between massless $U(1) \mathrm{s}$ ? 11

3. Supersymmetric models $\quad 12$

$\begin{array}{lll}\text { 4. The supergravity calculation of kinetic-mixing } & \mathbf{1 7}\end{array}$

$\begin{array}{lll}4.1 \text { A simple case without fluxes } & 18\end{array}$

$\begin{array}{ll}4.2 & \text { Inclusion of vacuum expectation values for fluxes }\end{array}$

5. Randall-Sundrum models $\quad 22$

$\begin{array}{lll}5.1 & \text { Green functions in one dimension } & 23\end{array}$

6. Kinetic mixing on the Klebanov-Tseytlin throat 25

$\begin{array}{ll}\text { 7. Conclusions } & 28\end{array}$

$\begin{array}{ll}\text { A. Remarks on the D3-Dי3 system } & 28\end{array}$

\section{Introduction}

Many extensions of the standard model (SM) contain hidden sectors that have no renormalizable interactions with SM particles. Notably, realistic embeddings of the standard model in $E_{8} \times E_{8}$ heterotic closed string theory as well as in type I, IIA, or IIB open string theory with branes, often require the existence of hidden sectors for consistency and for supersymmetry breaking ${ }^{1}$.

At the quantum level, hidden-sector particles will interact with SM particles through the exchange of massive messengers that couple to both the hidden and visible sectors, and this can lead to detectable traces of hidden sector physics. A unique window to hidden sectors is provided by hidden Abelian gauge bosons. In fact, hidden sector gauge groups

\footnotetext{
${ }^{1}$ For reviews which emphasize the occurence of hidden sectors in the context of string phenomenology, see e.g. Refs. [1, 2, 3, 4].
} 
often contain $U(1)$ gauge factors which generically mix kinetically $[5,6]$ with the hypercharge $U(1)$ of the visible sector, leading to terms in the low-energy effective Lagrangian of the form

$$
\mathcal{L} \supset-\frac{1}{4 g_{a}^{2}} F_{\mu \nu}^{(a)} F_{(a)}^{\mu \nu}-\frac{1}{4 g_{b}^{2}} F_{\mu \nu}^{(b)} F_{(b)}^{\mu \nu}+\frac{\chi_{a b}}{2 g_{a} g_{b}} F_{\mu \nu}^{(a)} F^{(b) \mu \nu}+m_{a b}^{2} A_{\mu}^{(a)} A^{(b) \mu},
$$

where $a(b)$ labels the visible (hidden) $U(1)$, with field strength $F_{\mu \nu}^{(a(b))}$ and gauge coupling $g_{a(b)}$. The dimensionless kinetic mixing parameter $\chi_{a b}$, appearing in front of the effective renormalizable operator in Eq. (1.1), can be generated at an arbitrarily high energy scale and does not suffer from any kind of mass suppression from the messengers that induce it. This makes it an extremely powerful probe of high scale physics; its measurement could provide clues to physics at energies that may never be accessible to colliders.

The mass mixing term $m_{a b}^{2}$ in Eq. (1.1) is, in the context of string theory, usually associated with the Stückelberg mechanism of mass generation for anomalous $U(1)$ s (see, e.g., Refs. $[7,8,9,10,11])$. The $m_{a b}^{2}$ effects were examined recently in the framework of the "Stückelberg $Z^{\prime}$ model" in $[12,13,14]$ whereby a massive (typically $\mathcal{O}(\mathrm{TeV})$ ) boson (which may also kinetically mix with the hypercharge) couples to the standard model particles directly via such a mass mixing, allowing it to be produced at the LHC; the large mass accounts for its current invisibility (see also Refs. [15, 16, 17, 18]). It is certainly a very plausible string-inspired model (possibly even a prediction).

Here, following our earlier work [19, 20], we will address the effect and the generation of the kinetic mixing term $\chi_{a b}$. We shall propose searching for truly hidden gauge fields which are anomaly-free and massless. In the presence of light or massless hidden fermions, this may be detected thanks to the kinetic mixing generated at loop level. This is a complementary string-motivated scenario, potentially providing different information about the compact space of string theory which may be impossible to ever obtain directly. An exhaustive study of the predicted size of kinetic mixing in realistic compactifications of heterotic string theory has been performed in Ref. [21]. Type II models were considered in previous work $[19,20]$ where we examined the mixing in non-supersymmetric string set-ups between branes and antibranes in large toroidal volumes and suggested that the non-observation of kinetic mixing may be able to place bounds on the string scale in more general scenarios, or alternatively may place a lower bound on the kinetic mixing to be observed based on the currently favoured string scale. However a systematic and rigorous study in the context of type II string models is still lacking, and this is the goal the present paper pursues.

Why would one expect kinetic mixing to be of interest in the context of type II models? Kinetic mixing appears in a Lagrangian when massive modes coupling to different $U(1) \mathrm{s}$ are integrated out $[5,22]$. In the type II context, hidden $U(1) \mathrm{s}$ arise as D-branes in the bulk that have no intersection with the branes responsible for the visible sector. The heavy modes that are integrated out correspond to open strings stretched between the visible and hidden stacks of branes. This can also be understood in the closed string channel as mediation by light or massless closed string (i.e. bulk) modes. The motivation for a comprehensive study in type II theories therefore derives from the following general 
observations: in type II string compactifications, hidden $U(1)$ s are ubiquitous, and there is no reason to expect all of them to be anomalous and hence heavy. Furthermore, the Ramond sector on intersecting D-branes always yields the massless charged matter fermions that could make the kinetic mixing detectable.

The type II models can be further subdivided into two classes depending on how curved the compact space is supposed to be. First there are models in which the compact space plays the role of a large quasi-flat bulk volume. These include the D-branes at singularities (so called bottom-up) models [23]. For the sake of simplicity we will also place within this class models in which intersecting D6-branes wrap 3-cycles on toroidal backgrounds [24], despite the volumes in this case being restricted to be rather small. The second class of models are those in which the compact volume is significantly warped and RandallSundrum [25, 26] like. In this class of models, which includes the KKLT scenario [27, 28], the standard model branes are typically assumed to be located at the bottom of a warped throat. Hidden branes may be present for a variety of reasons, such as tadpole and/or anomaly cancellation in the former class, or "uplift" in KKLT scenarios.

In the present paper, we shall extend the discussion of Refs. [19, 20] to consider set-ups in both of these categories, involving supersymmetric and nonsupersymmetric configurations of D-branes, both in large volumes and in warped backgrounds with fluxes. Our analysis (beginning in the following section) will demonstrate that kinetic mixing between visible and massless hidden $U(1) \mathrm{s}$ is an interesting possibility to search for in forthcoming experiments. Clearly the issue of Stückelberg masses and kinetic mixing are related, so one of the main aims of this paper will be to show how to disentangle them in the string calculation. We will show using a variety of methods how both the kinetic mixing and the Stückelberg mass mixing can be derived from the underlying type II string compactification. We will demonstrate by explicit example that kinetic mixing can occur without Stückelberg masses in a completely supersymmetric set-up where we can use conformal field theory (CFT) techniques. We will then develop a supergravity approach which allows us to examine the phenomenon in more general backgrounds, where we find that kinetic mixing is natural in the context of flux compactifications.

\subsection{Review: detection of hidden-sector $U(1)$ s and current limits}

Before beginning the analysis, we would like to review the possible methods of detection of hidden-sector $U(1)$ s, and the current observational limits. The masses of the hidden-sector photons and matter, and the kinetic mixing all come in to play, and because of this we will here give as general a discussion as possible, in particular elucidating the experimental differences in the possible detection of massless versus massive hidden $U(1)$ s. Indeed, the best way to search directly for the hidden-sector $U(1)$ gauge boson $\left(\gamma^{\prime}\right)$ depends primarily on its hitherto undetermined mass. For a mass in the range $m_{Z} \approx 100 \mathrm{GeV} \lesssim m_{\gamma^{\prime}} \lesssim 1 \mathrm{TeV}$, precision electroweak tests can be used $[15,16]$ to set an upper limit $\chi \lesssim$ few $\times 10^{-2}$ on the mixing parameter which will be only mildly improved by future measurements at the high-energy frontier by LHC and ILC [17, 18, 14]. For smaller masses, the best limits arise from searches for $\gamma \leftrightarrow \gamma^{\prime}$ oscillations [29, 30, 31, 32, 33] and for deviations from Coulomb's law (cf. Fig. 1). Note however that, if the hidden-sector $U(1)$ photons are massless (i.e. the 


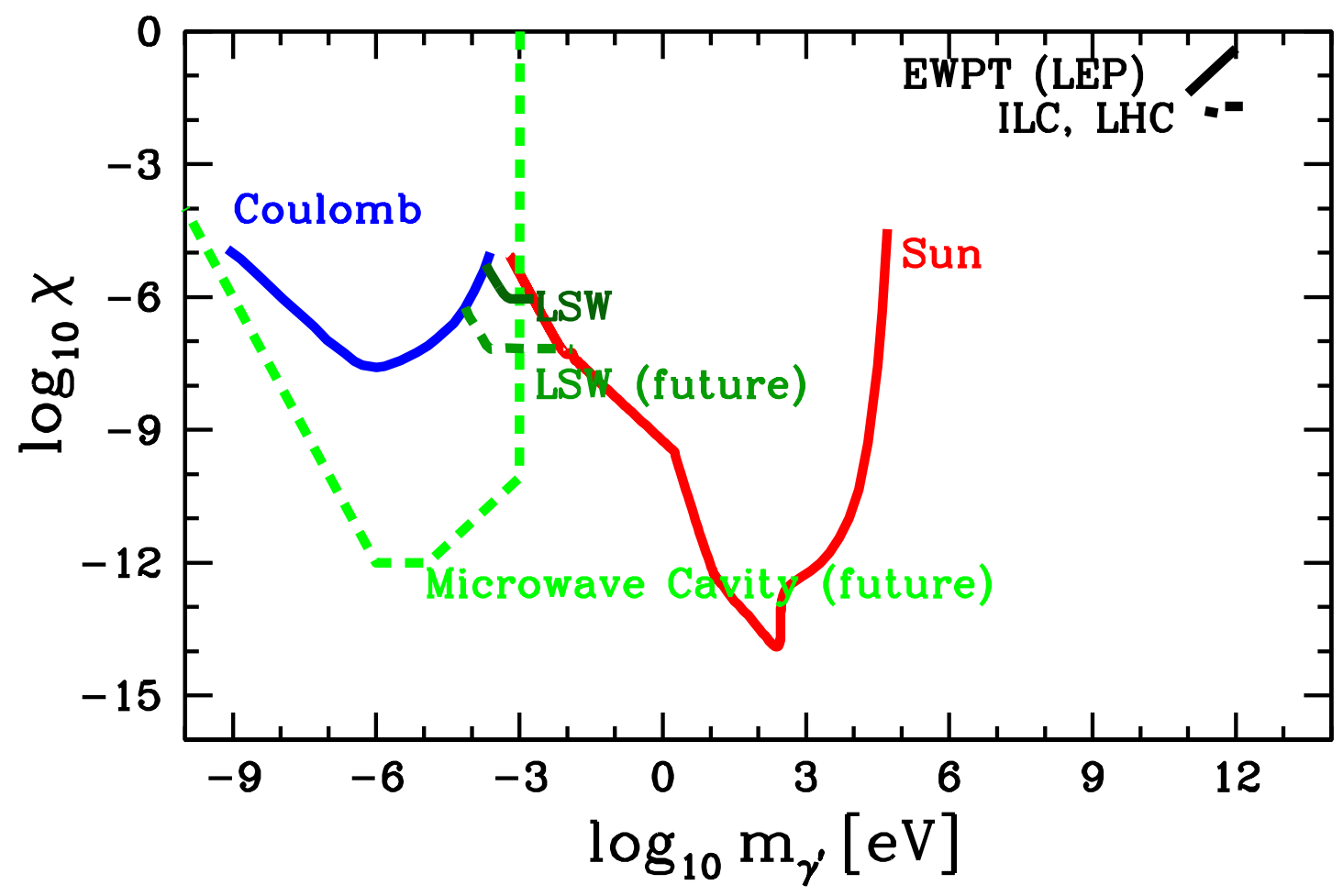

Figure 1: Upper limits on the kinetic mixing parameter $\chi$ versus the hidden-sector $U(1)$ gauge boson mass $m_{\gamma^{\prime}}$, from electroweak precision tests (EWPT) at LEP and future experiments at LHC and ILC [17, 18, 14], from searches for deviations of the Coulomb law [34, 35], and from searches for signatures of $\gamma \leftrightarrow \gamma^{\prime}$ oscillations, exploiting, as a photon source, current and future laboratory lasers (light-shining-through-a-wall (LSW) experiments) [30], future microwave cavities [31], or the sun $[32,33]$.

gauge symmetry is unbroken), then in the absence of light hidden matter there is no limit on its mixing with hypercharge (because the effect can be reabsorbed by a redefinition of the hypercharge coupling constant).

This is different if, in addition to the possibly light hidden-sector $U(1)$ gauge bosons, there are light hidden-sector matter particles which are charged under the hidden-sector $U(1)$ gauge symmetry. These could include for example a hidden-sector fermion $h$ with a bare coupling to $A_{\mu}^{(b)}$ given by

$$
\mathcal{L} \supset \bar{h} A^{(b)} h .
$$

Such particles are known to show up as electrically minicharged particles, with their electric charge being proportional to the gauge kinetic mixing parameter [5]. Indeed, upon diagonalizing the gauge kinetic term in Eq. (1.1) by the shift

$$
A_{\mu}^{(b)} \rightarrow \tilde{A}_{\mu}^{(b)}+\chi A_{\mu}^{(a)},
$$

the coupling term (1.2) gives rise to a coupling with the visible gauge field $A_{\mu}^{(a)}$,

$$
\bar{h} A^{(b)} h \rightarrow \bar{h} \tilde{A}^{(b)} h+\chi \bar{h} A^{(a)} h,
$$




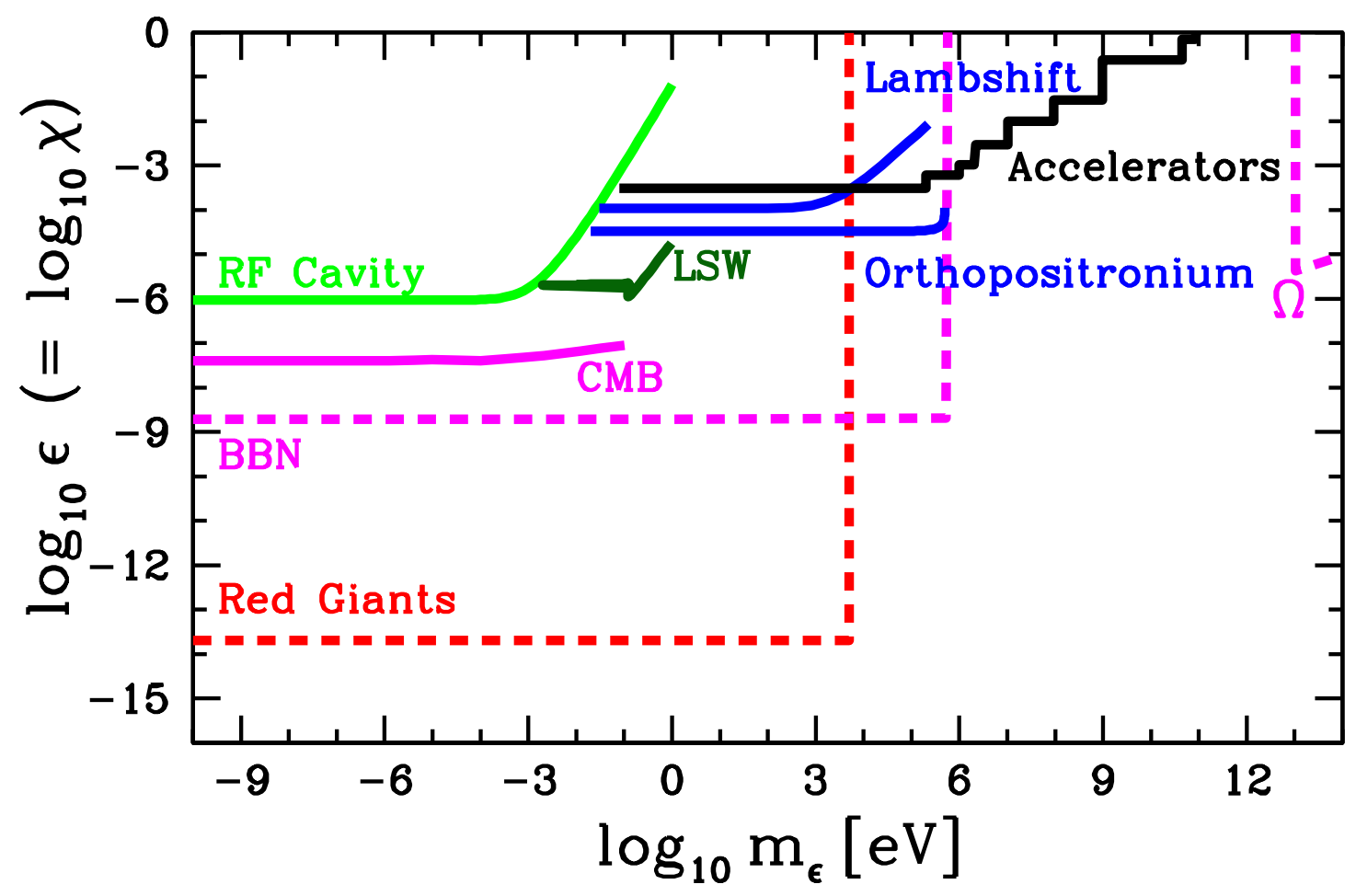

Figure 2: Upper limits on the fractional charge $\epsilon=Q_{\epsilon} / e$ of a hidden-sector fermion with mass $m_{\epsilon}$. Some of the limits only apply if there is also an ultralight hidden-sector $U(1)$ gauge boson which gives rise to the minicharge $\epsilon \sim \chi$ by gauge kinetic mixing with the photon. Laboratory limits arise from laser polarization and light-shining-through-a-wall (LSW) experiments [30], from energy loss considerations of RF cavities [36], from searches for the invisible decay of orthopositronium [37], from Lamb shift measurements [38] and from searches at accelerators [39, 40]. Limits from cosmology are due the non-observation of a significant distortion of the spectrum of the cosmic microwave background (CMB) radiation [41] (for a limit exploiting the CMB anisotropy, see Ref. [42]), due to the apparent successfullness of standard big bang nucleosynthesis (BBN) [43], and due to the observational requirement that the contribution of MCPs to the energy density should not overclose the universe, $\Omega=\rho / \rho_{\text {crit }}<1$ [44]. Finally, an astrophysical limit can be placed by energy loss considerations of red giants [43].

corresponding to a possibly small, non-integer charge with respect to the visible sector $U(1)$

$$
Q_{h}^{(a)}=\chi g_{b} \equiv \epsilon e .
$$

Hence in a wide class of models one can also look experimentally for signatures of the virtual or actual presence of electrically minicharged particles (MCPs). For low MCP masses, $m_{\epsilon} \lesssim 0.1 \mathrm{eV}$, the best current laboratory limits on the electric charge (cf. Fig. 2), $\epsilon \lesssim$ few $\times 10^{-7}[30]^{2}$, are obtained from laser polarization experiments [45, 46], such as BFRT [48], PVLAS [49, 50], and Q\&A [51], where linearly polarized laser light is sent through a transverse magnetic field, and changes in the polarization state are searched

\footnotetext{
${ }^{2}$ If there are hidden-sector photons in addition to the MCPs this bound may be somewhat weakened. The most robust bound then comes from light-shining-through-a-wall experiments discussed below [47].
} 
for. Such changes would signal that some photons are being retarded by interactions with virtual hidden-sector matter (the SM effect being too small to observe), or are being lost by pair production of hidden-sector matter particles. Other laser experiments, exploiting a light-shining-through-a-wall technique, such as ALPS [52], BFRT [53], BMV [54], GammeV [55], LIPSS [56], OSQAR [57], and PVLAS [58] are sensitive to $\gamma \leftrightarrow \gamma^{\prime}$ oscillations which can be induced, even for massless $\gamma^{\prime}$ s, by the presence of virtual hidden-sector matter in a magnetized vacuum [47]; current LSW data provide a limit of $\chi=\epsilon \lesssim 2 \times 10^{-6}$, for $m_{\gamma^{\prime}}=0$ and $m_{\epsilon} \lesssim 0.1 \mathrm{eV}$ [30]. A comparable laboratory limit, $\epsilon \lesssim 10^{-6}$, for $m_{\epsilon} \lesssim 1 \mathrm{meV}$, can be inferred from the non-observation of an excessive energy loss due to Schwinger pair production of minicharged particles in the strong electric fields in superconducting accelerator cavities [36]. In the mass range from $\mathrm{eV}$ up to the electron mass, the best laboratory limits, $\epsilon \lesssim 3 \times 10^{-5}$, arise from searches for the invisible decay of orthopositronium [37], while in the higher mass range the accelerator limits dominate; these, however, are rather loose (cf. Fig. 2 and Refs. [39, 40]). Bounds involving cosmology or astrophysics are seemingly much better, notably in the sub-electron mass region (cf. Fig. 2). However these limits, in particular those arising from BBN and energy loss constraints from red giants, are more model-dependent and can be considerably milder in certain parameter ranges of hidden-sector particles and interactions [59, 60, 61].

It is therefore reasonable to suppose that current and near future laser experiments have the potential to detect the presence of a hidden massless or light $U(1)$ gauge field coupled to charged hidden light $(\lesssim \mathcal{O}(\mathrm{eV}))$ matter. There is nothing to forbid these in the hiddensectors of type II string theory; assuming a collection of intersecting branes at some location of the compact space removed from the visible sector, there will be $U(1)$ factors of various masses and initially massless chiral multiplets. After supersymmetry breaking (presumably gravity-mediated) the charged bosons acquire masses, leaving massless fermions that we shall be interested in probing for; the charged bosons, we shall assume, acquire similar masses to their visible counterparts and are thus unobservable by the laser experiments previously mentioned. We shall thus assume no hidden Higgs mechanism acts to give masses to the fermions, although this could be relaxed provided the masses are sufficiently small.

\subsection{Overview: kinetic versus mass mixing in type II string theory}

Before getting to the details of the different scenarios, we should make some general remarks about kinetic mixing in string theory and outline the various computations we are going to perform. We will also at this point clarify the interplay between kinetic mixing, Stückelberg mass and anomaly cancellation.

Kinetic mixing can be understood in two ways: either as open strings stretching between separated branes, or as closed strings propagating between them. In principle, a string CFT computation can give the mixing, as in the case of toroidal models [19]. However that approach is limited in the sense that it can only address what can be done using CFT: it can only be used in models with backgrounds that are orbifolds or orientifolds of tori. In order to give a more general discussion it is far more useful to develop the effective supergravity approach. This reproduces the dominant contributions to kinetic mixing 
when computed as tree-level closed string propagation in toroidal compactifications, and allows us to consider more general gravitational and/or flux backgrounds, depending on the scenario in question. What the second method sometimes lacks in rigor, it more than makes up for in generality.

We shall begin our discussion in earnest in the next section by considering kineticmixing in the context of D-brane models in type IIB string theory using the CFT approach. For example one can think of supersymmetric models based on an orbifolded torus with an additional orientifolding. These supersymmetric models, first discussed in Ref. [62], are based on networks of wrapped intersecting D6-branes. They are a good starting point because here calculations can be done using CFT, and this will help us to develop an intuition for when kinetic mixing will occur and when it will not. This is a delicate question because the kinetic-mixing diagram is also the diagram for the mass term mixing visible and hidden $U(1) \mathrm{s}$, and a single one-loop open string diagram contributes to both of the terms in the Lagrangian of the form

$$
m_{a b}^{2} A_{\mu}^{(a)} A^{(b) \mu}+\frac{\chi_{a b}}{2 g_{a} g_{b}} F_{\mu \nu}^{(a)} F^{(b) \mu \nu},
$$

where $m_{a b}$ is the aforementioned Stückelberg mass mixing, associated with anomalies and their cancellation via the Green-Schwarz mechanism (a discussion of which can be found in Refs. $[7,8,9,10,11])$. Massless $U(1)$ s must have zero mixing, $m_{a b}=0$, with all the other $U(1) \mathrm{s}$ in the theory ${ }^{3}$. Since both of the terms in Eq. (1.6) arise from the same diagrams, how can $\chi_{a b}$ be non-vanishing between two anomaly-free $U(1) \mathrm{s}$ when we must also have $m_{a b}=0$ ?

The answer is that, in order to get a contribution to the Stückelberg mass, one has to extract a $1 / k^{2}$ pole from the appropriate one-loop integral (see Eq. (2.4) below). ¿From the closed string point of view this corresponds to the Stückelberg mass only getting contributions from massless closed string modes. Such contributions are blind to the location in the compact dimensions of the different sources. The non-pole contributions in this integral give rise to $\chi_{a b}$. Importantly these contributions to $\chi_{a b}$ are from both massless and massive Kaluza Klein modes. The latter certainly do care about the location of the sources in the compact dimensions, and so contributions to $\chi_{a b}$ do not generally cancel even when the contributions to $m_{a b} \mathrm{do}^{4}$. A schematic example is shown in Fig. 3. The picture indicates a localized standard model visible sector (on D6-branes, although the dimensionality is irrelevant) and a hidden sector $U(1)$ living on a brane together with the image brane in an orientifold plane. The contributions from the brane to the Stückelberg mass mixing between hidden and visible photons cancels that from its image. The same cancellation does not occur for kinetic mixing, because the hidden brane and its image are separated.

\footnotetext{
${ }^{3}$ Note that the converse is not true: absence of $4 \mathrm{~d}$ anomalies does not guarantee absence of Stückelberg masses because of possible $6 \mathrm{~d}$ anomalies. Also mass mixing between $\mathrm{U}(1) \mathrm{s}$ has been proposed in string models as a means of supersymmetry-breaking mediation [63], but we will not consider this possibility here.

${ }^{4}$ From a more field theoretic perspective we can argue as follows. Stückelberg masses typically arise from anomalies. Anomalies, however, do not care about the masses of the particles, i.e. the length of the stretched open strings. In contrast, kinetic mixing depends on the masses of the particles going around the loop.
} 


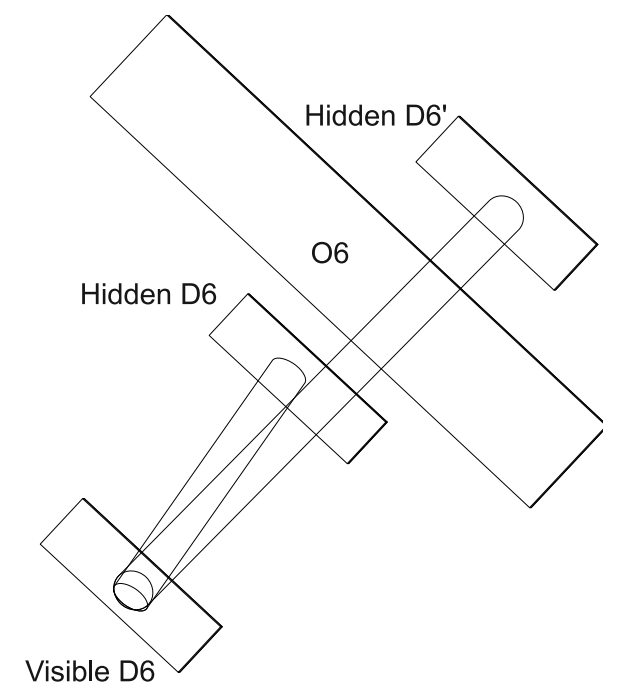

Figure 3: Schematic illustration of the reason why kinetic mixing need not cancel between anomalyfree $U(1) \mathrm{s}$. We show contributions to photon mixing with hidden $U(1) \mathrm{s}$ in the presence of an orientifold plane: Stückelberg mass-terms cancel, whereas kinetic mixing terms do not.

To demonstrate the validity of this general idea, in section 3 we will explicitly compute kinetic mixing in a supersymmetric and tadpole-free construction on a toroidal background, where we can calculate it with a straightforward CFT treatment. If kinetic mixing occurs between anomaly-free $U(1) \mathrm{s}$ here, then we can be sure that it can be decoupled from the question of anomaly cancellation. We begin in section 2 with the generalities of the CFT calculation. Then, in section 3, we present an anomaly-free toy model similar to those of Refs. [62, 24], but of course configured so as to have additional anomaly-free hidden $U(1)$ s.

Following that, in section 4, we will demonstrate that the same result can be readily computed using the effective supergravity field theory. As one might expect, the supergravity approach gives a more intuitive and general understanding which can then be applied to alternative scenarios, where the global properties of the models are not so well understood. In section 5, we consider a version of the Randall-Sundrum set-up which mimics the effect of warping. ¿From this we learn that kinetic mixing can be large in such models and is only tamed by fluxes generating sufficiently large masses for the mediating closed string fields. In particular the warping of the metric itself has no effect on the size of the kinetic mixing. We then confirm this in section 6 by considering the more stringy set-up of $U(1) \mathrm{s}$ located at the tip of a Klebanov-Tseytlin throat.

\section{The CFT computation of kinetic mixing: generalities}

We begin by reconsidering kinetic mixing in flat backgrounds where we can use CFT. Technically the computation is identical to finding gauge threshold corrections (cf. also Refs. $[64,65,66])$, but with a trivial but crucial difference: the group-theoretical prefactors 
are changed. Typically, an anomaly-free $U(1)$ is composed of a linear combination of the $U(1)$ s coming from different stacks of branes. The different $U(1)$ s will be labelled $a, b$ and the stacks will get labels $i, j^{5}$. The vertex operator describing $U(1)^{a}$ is given by

$$
V^{a}=\sum_{i} c_{i}^{a} V_{i}^{a}
$$

where we sum over stacks $i$ and the constants $c_{i}^{a}$ are chosen so that the corresponding $U(1)$ is anomaly free. The individual vertex operator on a given stack of branes $i$ is given by (in the zero-picture)

$$
V_{i}^{a}=\lambda_{i}^{a} \varepsilon_{\mu}\left(\partial X^{\mu}+2 \alpha^{\prime}(k \cdot \psi) \psi^{\mu}\right) e^{\mathrm{i} k \cdot X},
$$

where as usual $\varepsilon_{\mu}$ is the polarization vector, $\psi^{\mu}$ and $X^{\mu}$ are worldsheet fermions and bosons respectively and $\lambda_{i}^{a}$ is the Chan-Paton matrix on the stack $i$. The anomaly free $U(1)$ s are the linear combinations that obey

$$
\sum_{i} c_{i}^{a} \operatorname{tr}_{i}\left(\lambda_{i}^{a}\right)=0
$$

The general expression for the amplitude we calculate is then (in the closed string channel) $[7,19]$

$$
\begin{array}{r}
\left\langle V_{i}^{a} V_{j}^{b}\right\rangle=4\left(\alpha^{\prime}\right)^{2} \operatorname{tr}_{i}\left(\lambda_{i}^{a}\right) \operatorname{tr}_{j}\left(\lambda_{j}^{b}\right) \varepsilon_{\mu} \varepsilon_{\nu}\left(g^{\mu \nu} k^{2}-k^{\mu} k^{\nu}\right) \int_{0}^{\infty} d l \int_{0}^{1} d x e^{k^{\mu} k^{\nu} G_{\mu \nu}} \\
\sum_{\nu}\left[\frac{\theta_{4}^{\prime \prime}(x)}{\theta_{4}(x)}-\frac{\theta_{\nu}^{\prime \prime}(0)}{\theta_{\nu}(0)}\right] \frac{1}{\left(8 \pi^{2} \alpha^{\prime}\right)^{2}} \frac{\theta_{\nu}(0)}{\eta^{3}(\mathrm{i} l)} Z_{\nu}^{i j}(\mathrm{i} l),
\end{array}
$$

where the Green function is given by

$$
G_{\mu \nu}(x)=-2 \alpha^{\prime} g_{\mu \nu} \log \left|\frac{1}{l} \frac{\theta_{4}(x)}{\eta^{3}(\mathrm{i} l)}\right|,
$$

$\theta$ and $\eta$ are the elliptic theta and Dedekind eta functions, and $\operatorname{tr}_{i}, \operatorname{tr}_{j}$ denote that the trace is to be taken over the individual branes inside a stack $i$ or $j . Z_{\nu}^{i j}$ is the partition function with spin structure $\nu$, with the $i j$ indices indicating that it is in general a function of the displacements between the stacks. In the open string channel, this is a non-planar diagram (i.e. with the two vertex operators placed on different boundary stacks).

The final result for kinetic mixing between say hypercharge $A_{\mu}^{a}$ and a hidden anomalyfree $U(1) A_{\mu}^{b}$ contains a further $i, j$ sum over all the relevant stacks contributing to each $U(1)$ as dictated by Eq. (2.1). Note that if we are considering an orbifold model with fractional branes where the branes are separated, only the non-fractional component contributes: there can be no contribution from twisted sectors because a displacement between the ends of the annulus diagram is not consistent with an orbifold twist.

The form for the amplitude in the on-shell/low-energy limit $k^{2} \rightarrow 0$ will then be

$$
\left\langle V^{a} V^{b}\right\rangle=m_{a b}^{2} A_{\mu}^{a} A_{b}^{\mu}+\frac{\chi_{a b}}{g_{a} g_{b}} k^{2} A_{\mu}^{a} A_{b}^{\mu} .
$$

\footnotetext{
${ }^{5}$ When orientifolds are present the images are counted as different stacks.
} 
Since the right hand side of Eq. (2.4) explicitly contains the transverse structure $k^{2} g^{\mu \nu}-k^{\mu} k^{\nu}$, the contribution to the mass must come from a $1 / k^{2}$ pole of the integral. To make the pole structure manifest we take the large $l$ limit,

$$
e^{k^{\mu} k^{\nu} G_{\mu \nu}}=e^{-\frac{1}{4} 2 \pi \alpha^{\prime} k^{2} l},
$$

while the rest of the integrand can be generically expanded as

$$
\sum_{\nu}\left[\frac{\theta_{4}^{\prime \prime}(x)}{\theta_{4}(x)}-\frac{\theta_{\nu}^{\prime \prime}(0)}{\theta_{\nu}(0)}\right] \frac{1}{\left(8 \pi^{2} \alpha^{\prime}\right)^{2}} \frac{\theta_{\nu}(0)}{\eta^{3}(\mathrm{i} l)} Z_{\nu}^{i j}(\mathrm{il}) \propto 1+\sum_{\beta_{i j}>0} N\left(\beta_{i j}\right) e^{-\pi \beta_{i j} l},
$$

where $N(\beta)$ counts the multiplicity of closed-string modes at level $\beta$ (which includes KaluzaKlein and winding modes). The first term corresponds to massless closed string states and results in a $1 / k^{2}$ pole, generating a mass-like term for the gauge fields. This term is $i, j$ independent. On the other hand, $\chi_{a b}$ receives contributions from the second term which does depend on the displacement between $i$ and $j$.

As we have already stated, the sum of all contributions to the mass-term must be absent between two anomaly-free gauge groups. These have been calculated in, for example, Ref. [7], where the role of the mass-mixing term is elucidated as the Stückelberg mass generated for anomalous gauge groups, which emerges when the gauge field "eats" the relevant closed string modes. (In the case of D3-branes the modes that are eaten are NSNS $B_{2}$ fields and R-R $C_{2}$ fields, as will become apparent later.) At the same time, the kinetic mixing parameter $\chi$ gets contributions from both the pole and the non-pole parts of the integrand, with the latter corresponding to massive intermediate states with $\beta \neq 0$.

To give a specific illustration, consider the example of a single D3 brane and a $\bar{D} 3$ brane on a $T^{6}$ factorized into 3 complex 2 -tori labelled by $\kappa=1 \ldots 3$ :

$Z_{\nu}^{i j}(\mathrm{i} l)=\frac{1}{2} \delta_{\nu}^{\prime} \frac{\left(\alpha^{\prime}\right)^{3}(2 \pi)^{6}}{8 V_{6}} \frac{\theta_{\nu}^{3}(0)}{\eta^{9}(\mathrm{i} l)} \prod_{\kappa} \sum_{q^{\kappa}, p^{\kappa}} \exp \left[-\frac{\pi \alpha^{\prime} l}{2 T_{2}^{\kappa} U_{2}^{\kappa}}\left|q^{\kappa}+\bar{U}^{\kappa} p^{\kappa}\right|^{2}-\frac{2 \pi \mathrm{i}}{U_{2}^{\kappa}} \operatorname{Im}\left(z_{i j}^{\kappa}\left(\bar{U}^{\kappa} p^{\kappa}+q^{\kappa}\right)\right)\right]$

where $U^{\kappa}, T^{\kappa}$ are the complex and Kähler moduli, and $z_{i j}^{\kappa}$ is the complex separation vector between branes $i$ and $j$ (scaled to be dimensionless; the true distance is $2 \pi z_{i j}^{\kappa} \sqrt{T_{2}^{\kappa} / U_{2}^{\kappa}}$ ). The above contains the expected massless mode whose effect can be seen in the $l \rightarrow \infty$ limit:

$$
\begin{gathered}
\left\langle V_{i}^{a} V_{j}^{b}\right\rangle=\operatorname{tr}_{i}\left(\lambda_{i}^{a}\right) \operatorname{tr}_{j}\left(\lambda_{j}^{b}\right) \varepsilon_{\mu} \varepsilon_{\nu}\left(g^{\mu \nu} k^{2}-k^{\mu} k^{\nu}\right) \int_{0}^{\infty} d l \frac{\left(2 \pi \alpha^{\prime}\right)^{4}}{4 \alpha^{\prime} V_{6}} e^{-\frac{1}{4} 2 \pi \alpha^{\prime} k^{2} l} \\
\left\{1+\prod_{\kappa} \sum_{q^{\kappa}, p^{\kappa} \neq 0} \exp \left[-\frac{\pi \alpha^{\prime} l}{2 T_{2}^{\kappa} U_{2}^{\kappa}}\left|q^{\kappa}+\bar{U}^{\kappa} p^{\kappa}\right|^{2}-\frac{2 \pi \mathrm{i}}{U_{2}^{\kappa}} \operatorname{Im}\left(z_{i j}^{\kappa}\left(\bar{U}^{\kappa} p^{\kappa}+q^{\kappa}\right)\right)\right]\right\}(1+\text { string mass terms }) .
\end{gathered}
$$

We can see explicitly that only the first term, 1 , in the curly brackets gives a $z_{i j}$ independent contribution. The second term depends explicitly on the positions $z_{i j}$. Since it corresponds to massive closed string modes it contributes only to the kinetic mixing.

The reason for neglecting terms of order the string mass in (2.10) is the familiar exponential damping of massive modes beyond their wavelength; the phase factor in the 
above ensures that it is an accurate approximation to consider only the Kaluza-Klein expansion of the tori. This implies that it is the closed string modes which heavily dominate the process once the branes are separated by more than a string length, and moreover that we should be able very accurately to reproduce the expression above using only field theory as we shall do in section 4 .

The expected mass-mixing between the $U(1)_{a}$ of the D3, and the $U(1)_{b}$ of the $\overline{\mathrm{D}} 3$ coming from the massless modes is found to be

$$
\mathcal{S} \supset \int d^{4} x \frac{1}{\alpha^{\prime}} \frac{\left(2 \pi \alpha^{\prime}\right)^{3}}{V_{6}} A_{\mu}^{a} A^{b \mu} .
$$

Note that in the D3- $\overline{\mathrm{D}} 3$ system we also have a contribution from planar diagrams (i.e. with both vertex operators placed on the same boundary in contrast with Eqs. (2.4),(2.10)). This generates gauge threshold corrections but more importantly renders any gauge group carried by the brane or antibrane massive. This is a consequence of the uncancelled NS-NS charges, i.e. that there is a nonzero cosmological constant. Hence, due to the volume suppression of the masses, this could be a candidate for the Stückelberg $Z^{\prime}$ scenario; we make some remarks on this in appendix A. Moreover there is kinetic mixing. We obtain for $\chi$ in Eq. (1.1)

$$
\chi_{a b} \approx g_{a} g_{b} \frac{\left(2 \pi \alpha^{\prime}\right)^{3}}{V_{6}} \sum_{q^{\kappa}, p^{\kappa} \neq 0} \frac{\exp \left[\sum_{\kappa}-\frac{2 \pi \mathrm{i}}{U_{2}^{\kappa}} \operatorname{Im}\left(p^{\kappa} z^{\kappa} \bar{U}^{\kappa}+q^{\kappa} z^{\kappa}\right)\right]}{\sum_{\kappa} \frac{\alpha^{\prime}}{T_{2}^{\kappa} U_{2}^{\kappa}}\left|q^{\kappa}+\bar{U}^{\kappa} p^{\kappa}\right|^{2}}
$$

where $z^{k}$ is the displacement between the brane and anti-brane in the $k^{\prime}$ th complex 2-torus. Note that the kinetic-mixing term produced by the string amplitude, $\chi_{a b} / g_{a} g_{b}$, actually contains no factors of the gauge coupling (since the vertex operators carry none), and that in addition $\chi$ depends on $z^{k}$ but the mass-mixing does not.

\subsection{When can we have kinetic mixing between massless $U(1)$ s?}

We now wish to show that kinetic-mixing can occur between anomaly-free $U(1) \mathrm{s}$. To begin with, note that the amplitude is always proportional to the trace of the Chan-Paton factor for the $U(1)^{a}$ gauge factor $\operatorname{tr}_{i}\left(\lambda_{i}^{a}\right)$. This is also the factor for the mass-mixing term, and so if we wish to avoid a massive gauge field, the total contribution for gauge group a must vanish: if the $U(1)$ charge is given by a linear combination of the trace $U(1)$ charges on the branes as $Q^{a}=\sum c_{i}^{a} Q_{i}^{a}$, then we must have either

$$
\sum_{i} c_{i}^{a} \operatorname{tr}_{i}\left(\lambda_{i}^{a}\right)=0
$$

or its equivalent for the hidden sector $U(1)$ s. However if we are considering a $U(1)^{a}$ split among separate branes, we cannot simply factor out $\sum_{i} c_{i}^{a} \operatorname{tr}_{i}\left(\lambda_{i}^{a}\right)$ from the kinetic-mixing term because the integrand depends on the positions (on $z_{i j}^{k}$ in other words).

As an example if we have a $U(1)_{a}$ split among separate branes, this can mix with other $U(1)$ fields. This can also be understood in field theory as the embedding of the $U(1)$ as the generator of a broken non-Abelian group; for example for two branes carrying 
naively a $U(2)$ when coincident, upon splitting there are two $U(1)$ charges $Q_{1}$ and $Q_{2}$. The combination $\frac{Q_{1}}{2}+\frac{Q_{2}}{2}$ is anomalous, and corresponds to the $U(1)$ in $U(2)=U(1) \times S U(2)$, while the combination $\frac{Q_{1}}{2}-\frac{Q_{2}}{2}$ is always non-anomalous and corresponds to the $\sigma_{3}$ generator in $S U(2)$.

If there is an orientifolding (which is generally required in order to cancel tadpoles), then it is typically accompanied by a reflection $R$ on the compactified coordinates which generates orientifold planes and D-brane images. Then we automatically have pairs of branes. Under the orientifold action, each brane $i$ has an image $i^{\prime}$, which carries the same gauge group. The Chan-Paton matrices of the brane and its image are related by

$$
\lambda_{i}^{\prime}=\mp \gamma_{\Omega R}^{-1} \lambda_{i}^{T} \gamma_{\Omega R},
$$

where $\gamma_{\Omega R}$ determines the action of the orientifold on the Chan-Paton matrices, and where the minus (plus) sign is fixed by consistency of the model, and arises from the odd number of worldsheet fermions in physical states imposed by the GSO projection. Since the relevant charges come from $\operatorname{tr}\left(\lambda_{i}\right)$, however, the image branes have charges $Q_{i^{\prime}}=\mp Q_{i}$. For such branes sitting on the orientifold we find a $U S p(2 N)(S O(2 N))$ gauge group, for $N$ branes and $N$ images, and note that the trace generator is in the former case projected out. When the branes are separated from the orientifold plane, however, while remaining in the same homology class $^{6}$ we automatically have a non-anomalous $U(1)$. Again this non-anomalous $U(1)$ can be thought of as coming from a traceless generator of the symplectic group when the branes are on top of the orientifold plane. Indeed, in the presence of an orientifold plane, the mass-mixing between the $U(1)_{i}$ coming from the hidden brane gets contributions from the brane and its image, which together will be proportional to $\operatorname{tr}_{i}\left(\lambda_{i}\right)+\operatorname{tr}_{i}\left(\lambda_{i}^{\prime}\right)=0$. Notice that this is independent of whether the visible $U(1)$ in question is itself anomalous: the $U(1)$ from an isolated D-brane does not get masses from any source if it is parallel to an orientifold plane, but since the brane and its image are displaced it can still kinetically mix. In fact, in a large volume compactification, the orientifold and the image can be removed to large distances and the resulting kinetic-mixing would be dominated by the single brane. In the closed string picture, the orientifolding projects out the massless modes that transmit the mass-mixing. A general illustration of this scenario is given in figure 3 .

\section{Supersymmetric models}

In order to confirm that kinetic mixing can indeed occur between anomaly free and massless $U(1)$ s we will now, as promised in the Introduction, examine self-consistent (i.e. tadpolefree) global configurations that have non-vanishing kinetic mixing between mutually supersymmetric branes.

A convenient framework in which to construct supersymmetric models consists of a simple orientifold with D6 branes and O6 planes in type IIA string theory, as reviewed

\footnotetext{
${ }^{6}$ Such as where there are translation or Wilson line moduli, or in a separate region of a Calabi-Yau related to the original by an involution of the compact space. It is important that the quantum states be related, otherwise the contributions to the gauge field mass between brane and image will not be equal.
} 

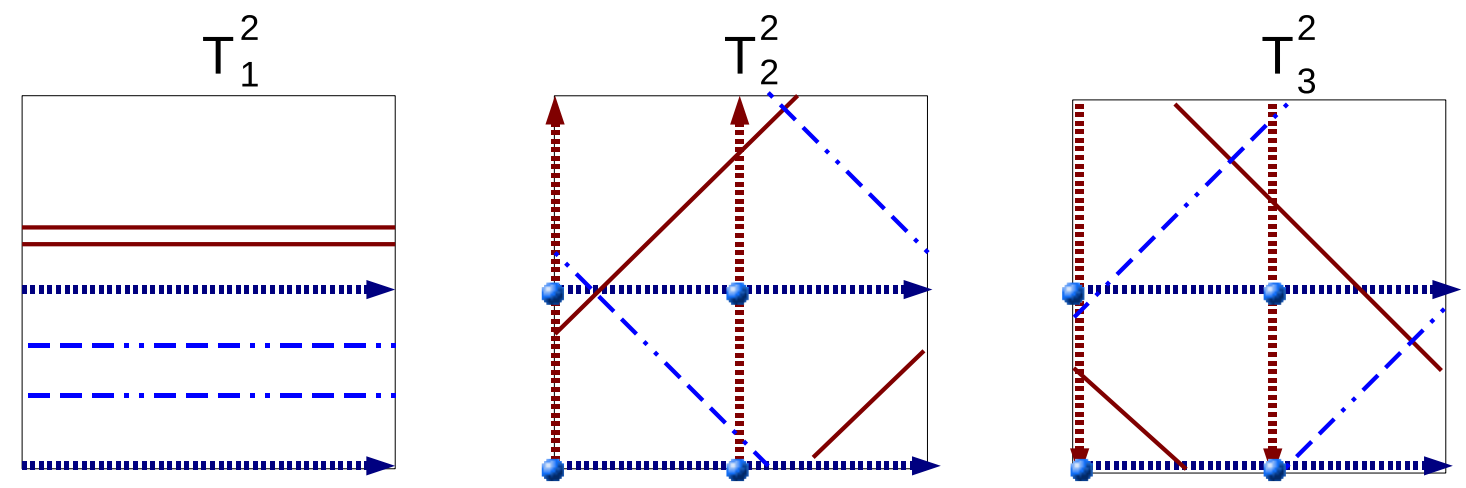

Figure 4: Supersymmetric configuration corresponding to the model in Table 1. Solid lines denote $A$ stacks and dashed-dotted lines represent $B$ stacks. Each of these stacks is separated into $A_{1}, A_{2}$, and $B_{1}, B_{2}$ in the first torus only. The orientifold planes are represented by the dashed lines with arrows. In the first torus, the two sets of orientifold planes are coincident. Finally the dots on each of the last two tori show the orbifold fixed points (or more precisely planes).

in Ref. [24]. In principle, one would like to construct an $\mathcal{N}=1$ model similar to those of Ref. [62], but with hidden $U(1)$ s. However the D6-branes wrap all the internal cycles and typically they always intersect. Hence it is difficult to construct $\mathcal{N}=1$ models with any hidden sector. Our main aim here is not to construct a realistic model, but rather to provide a simple proof of concept in a completely supersymmetric set-up, for which $\mathcal{N}=2$ models will be sufficient. Such models correspond to dimensionally reduced 6-dimensional $\mathcal{N}=1$ models, and so Stückelberg masses, if they are present, correspond to the GreenSchwarz anomaly cancellation of 6-dimensional anomalies (the 4-dimensional anomalies in $\mathcal{N}=2$ being of course zero).

Our general configuration is as follows: we choose the ten-dimensional spacetime to be $\mathbb{R}^{3,1} \times \mathbb{T}^{2} \times\left(\mathbb{T}^{2} \times \mathbb{T}^{2}\right) / \mathbb{Z}_{2}$, where the $\mathbb{Z}_{2}$ orbifolding is taken to act on the second and third $\mathbb{T}^{2}$ tori. The tori are all taken to be rectangular. Denoting the complex coordinates on the compact space $z^{i} \in \mathbb{T}_{i}^{2}$, the orbifold involution acts as $\theta:\left(z_{2}, z_{3}\right) \rightarrow\left(-z_{2},-z_{3}\right)$. The orientifold involution is then introduced as follows. It consists of world sheet parity transformation $\Omega$ coupled with a non-holomorphic reflection $R$ in the internal complex cooordinates, $R: z^{k} \rightarrow \bar{z}^{k}$. The projections leave $4 \times 4=16$ fixed points of the $\mathbb{Z}_{2}$ orbifold (see Fig. 4), and 16 orientifold fixed planes (O6-planes), 8 for each of the orientifold actions, $\Omega R, \Omega R \theta$.

This orbifold is a singular limit of the Calabi-Yau manifold $\mathbb{T}^{2} \times K 3$. Although we can calculate exactly only in the orbifold limit, we can make completely general statements about how the D6-branes should wrap in order to obtain kinetic mixing: we must place our branes on bulk rather than exceptional cycles; the D6 branes must wrap two-cycles on the $K 3$, and a one-cycle on the $\mathbb{T}^{2}$; thus both the closed and open string sectors preserve $\mathcal{N}=2$ supersymmetry in four dimensions (such as prior to $D$-term SUSY-breaking in 


\begin{tabular}{|c||c|c|c||}
\hline stack & $N$ & gauge group & $\left(n^{1}, m^{1}\right) \times\left(n^{2}, m^{2}\right) \times\left(n^{3}, m^{3}\right)$ \\
\hline$A$ & $4+4$ & $S U(2) \times S U(2), Q_{A_{1}}, Q_{A_{2}}$ & $(1,0) \times(1,1) \times(1,-1)$ \\
$B$ & $4+4$ & $S U(2) \times S U(2), Q_{B_{1}}, Q_{B_{2}}$ & $(1,0) \times(1,-1) \times(1,1)$ \\
\hline
\end{tabular}

Table 1: Wrapping numbers for a very simple model with kinetic mixing. As usual, $N$ counts branes plus their orbifold images.

split-supersymmetry models $[67])^{7}$.

In the calculable orbifold case, we may derive rather general expressions for the subsequent kinetic mixing before presenting an explicit model (detailed in Table 1 and Fig. 4). We need only assume that the two massless gauge groups $U(1)_{a}$ and $U(1)_{b}$ come from two parallel stacks of branes each, labelled $A_{1}, A_{2}$ and $B_{1}, B_{2}$. In order to not intersect, they must be parallel to the orientifold plane in torus 1, but not lie upon it (cf. Fig. 4). We denote the separations from the O6-plane in the torus $1 y_{A_{i}}$, and write $\delta_{i j} \equiv y_{A_{i}}-y_{B_{j}}$. Note that it is possible to take $A_{2}=A_{1}^{\prime}$ (or similarly $B_{2}=B_{1}^{\prime}$ ), the image under the orientifold, but we shall not require this. The charges for the massless combinations are given by $Q_{a}=\frac{1}{N_{A_{1}}} Q_{A_{1}}-\frac{1}{N_{A_{2}}} Q_{A_{2}}=\sum_{i} \frac{c_{i}^{a}}{N_{A_{i}}} Q_{A_{i}}$, and similarly for $Q_{b}$, where $N_{A_{i}}$ is the number of branes in stack $A_{i}$, and $Q_{A_{i}}, Q_{B_{i}}= \pm 1$. The kinetic mixing is then given by

$$
\chi=\sum_{i j} c_{i}^{a} c_{j}^{b} Q_{A_{i}} Q_{B_{j}} \chi_{i j}=\chi_{11}-\chi_{12}-\chi_{21}+\chi_{22}
$$

where $[65,70]$

$$
\chi_{i j}=\frac{g_{a} g_{b}}{4 \pi^{2}} I_{A B}\left[\log \left|\frac{\theta_{1}\left(\frac{\mathrm{i} \delta_{i j} L_{1}}{2 \pi^{2} \alpha^{\prime}}, \frac{\mathrm{i} T_{1}^{2}}{\alpha^{\prime}}\right)}{\eta\left(\frac{\mathrm{i} T_{1}^{2}}{\alpha^{\prime}}\right)}\right|^{2}-\frac{\delta_{i j}^{2}}{2 \pi^{3} \alpha^{\prime}} \frac{\left(L_{1}\right)^{2}}{T_{2}^{1}}\right],
$$

where $\chi_{i j}$ is the kinetic mixing between $A_{i}$ and $B_{j}$; and $I_{A B}$ is the number of intersections between the branes in the non-parallel directions, $L_{1}$ is the length of both branes in the torus 1 in which they are parallel, and finally $T_{2}^{1}$ is the Kähler modulus of torus 1 , proportional to the product of the radii in the case of rectangular tori. Note that the above can also be calculated exactly by the effective supergravity techniques that will be introduced in the next section, since supersymmetry ensures that all of the string mass excitations do not contribute. Note also that it is crucial that the two stacks of branes preserve a mutual $\mathcal{N}=2$ supersymmetry; if they only preserved $\mathcal{N}=1$ supersymmetry there would be no dependence on the separation, and thus we could not separate mass mixing from kinetic mixing, and if they preserved $\mathcal{N}=4$ the amplitude would cancel. This is, however, merely a peculiarity of the very symmetric toroidal orientifold setup that we are using, and the fact that we are using D6-branes.

\footnotetext{
${ }^{7}$ Despite the fact that the kinetic mixing can be calculated exactly only in the orbifold limit case, these statements are valid more generally because the calculation of mixing in $\mathcal{N}=2$ depends only on the zero modes, with the massive string excitations cancelling $[68,69]$ - and thus depends only on the intersection form on the $K 3$.
} 
It is easy to see that for generic values of the brane positions, and in the presence of massless fermions, the induced mixing would violate the bounds on kinetic mixing by many orders of magnitude. This is because, with wrapped D6-branes, one is unable to take a large volume limit to try and dilute it, and in addition (when the cycles wrapped by the branes and the bulk radii are all of the same order) dilution only occurs for $p \leq 5$. It is possible to give a mixing of the order of $10^{-6}$ or less by tuning the configuration: suppose that $\left(y_{B_{1}}-y_{B_{2}}\right) \sim T_{1}^{2} / L_{1} \gg l_{s}>\left(y_{A_{1}}-y_{A_{2}}\right)$, i.e. the branes are placed generically but one splitting is much larger than the other, ensuring $\delta_{i j}>l_{s}$ (since for small $\delta_{i j}$ the mixing grows logarithmically). We then find

$$
\chi=\frac{g_{a} g_{b}}{4 \pi^{2}} I_{A B}\left[\frac{\left(y_{A_{1}}-y_{A_{2}}\right)\left(y_{B_{1}}-y_{B_{2}}\right)}{\pi^{3} \alpha^{\prime}} \frac{\left(L_{1}\right)^{2}}{T_{1}^{2}}\right]+O\left(e^{-\pi T_{1}^{2} / \alpha^{\prime}}\right) .
$$

and thus $\left(y_{A_{1}}-y_{A_{2}}\right) \sim 10^{-6} \frac{l_{s}^{2}}{L_{1}}$. Note that despite the fact that this distance is much smaller than the string scale, the expressions are still valid since they are derived from the complete CFT, and moreover such displacements are quite natural when considered from the field theory perspective since they represent a Higgsing of the gauge group by giving a vacuum expectation value to an adjoint scalar.

As an extremely simple, explicit, example, we present the wrapping numbers for two stacks of branes in table 1. The configuration including orientifold planes is shown in Fig. 4.

The model has to satisfy a number of consistency conditions. First to preserve supersymmetry, the radii of the tori are constrained. Denoting by $\theta_{i}$ the angle in the $i^{\text {th }}$ torus between the branes and the $\mathrm{O} 6_{\Omega}$-planes, we must have $\theta_{1}+\theta_{2}+\theta_{3}=0$; in the present $\mathcal{N}=2$ case we have $\theta_{1}=0$, which leads to

$$
U_{2}^{(2)} \frac{m_{2}}{n_{2}}+U_{2}^{(3)} \frac{m_{3}}{n_{3}}=0
$$

where in the rectangular case the complex structure moduli $U^{(i)}=\mathrm{i} U_{2}^{(i)}=\mathrm{i} R_{2}^{(i)} / R_{1}^{(i)}$ are simply the ratio of torus radii. This condition may trivially be satisfied provided $n_{i} / m_{i}$ is the same for all the branes up to an overall factor. The tadpole cancellation conditions that must be satisfied are as follows. First we ensure that the R-R (7-form) charge contribution from the orientifold planes cancels that of the D6-branes. The homology class of a brane $A$ with wrappings $\left(n^{i}, m^{i}\right)$ (where $i$ labels the tori) is $\left[\Pi_{A}\right]=\sum_{i=1}^{3} n^{i}\left[a_{i}\right]+m^{i}\left[b_{i}\right]$, where the canonical $\left[a_{i}\right]$ cycles correspond to the $\operatorname{Re}\left(z_{i}\right)$ coordinate, and the $\left[b_{i}\right]$ cycles to $\operatorname{Im}\left(z_{i}\right)$. The images under the orientifold have homology $\left[\Pi_{A^{\prime}}\right]=\sum_{i=1}^{3} n^{i}\left[a_{i}\right]-m^{i}\left[b_{i}\right]$. The O6planes corresponding to $\Omega R$ (henceforth denoted $06_{\Omega R}$ ), have wrapping numbers $(1,0) \times$ $(1,0) \times(1,0)$, and hence homology $\left[\Pi_{O_{\Omega R}}\right]=\left[a_{1}\right] \times\left[a_{2}\right] \times\left[a_{3}\right]$, while those corresponding to $\Omega R \theta$ (henceforth $\mathrm{O}_{\Omega R \theta}$ ), have wrapping numbers $(1,0) \times(0,1) \times(0,-1)$, and homology $\left[\Pi_{O_{\Omega R \theta}}\right]=-\left[a_{1}\right] \times\left[b_{2}\right] \times\left[b_{3}\right]$. Their D6 charges are -4 , so the tadpole cancellation condition is

$$
\sum_{A} N_{A}\left(\left[\Pi_{A}\right]+\left[\Pi_{A^{\prime}}\right]\right)=4 \times 8\left(\left[\Pi_{O_{\Omega R}}\right]+\left[\Pi_{O_{\Omega R \theta}}\right]\right)
$$


which, assuming all the branes have $m^{1}=0$, yields only two constraints on the wrapping numbers:

$$
\begin{gathered}
\sum_{A} N_{A} n_{A}^{1} n_{A}^{2} n_{A}^{3}=16, \\
\sum_{A} N_{A} n_{A}^{1} m_{A}^{2} m_{A}^{3}=-16 .
\end{gathered}
$$

These constraints are clearly satisfied by the model in table 1. (Supersymmetry then ensures the cancellation of the NS-NS tadpoles.)

Kinetic mixing arises when the stacks of branes are displaced from the $\mathrm{O} 6_{\Omega R}$-planes in the first torus. The counting goes as follows. Begin with $N$ branes plus their orientifold images on top of the orientifold plane, and passing through orbifold fixed points. As we move away from the fixed points/planes we get images under both the orbifold and the orientifold, so that we have a $U(N / 2)$ gauge group. By further splitting the stacks in the first torus, we obtain two separate $U(N / 4)$ gauge groups, and by taking the trace generator from each, we can form massless $U(1)$ combinations $Q_{a}=\frac{4}{N_{A}}\left(Q_{A_{1}}-Q_{A_{2}}\right), Q_{b}=$ $\frac{4}{N_{B}}\left(Q_{B_{1}}-Q_{B_{2}}\right)$ as described in section 2.1 (where 4 counts orbifold images). Note that Stückelberg masses arise for the orthogonal $U(1)$ combinations, $Q_{\bar{a}}=\frac{4}{N_{A}}\left(Q_{A_{1}}+Q_{A_{2}}\right)$ and $Q_{\bar{b}}=\frac{4}{N_{B}}\left(Q_{B_{1}}+Q_{B_{2}}\right)$, as expected.

The kinetic mixing for this particular model is given by $2 \chi$, with $\chi$ as in equation (3.3); note that there is no mixing between the branes and orientifold images, since $B^{\prime}$ is parallel to $A$ (and thus preserves a mutual $\mathcal{N}=4$ supersymmetry, cancelling any mixing), but the overall factor of 2 accounts for $\chi_{A_{1}^{\prime} B_{1}^{\prime}}=\chi_{A_{1} B_{1}}$ etc. The above expression will be non-zero provided that the $y$ 's are not equal.

We can straightforwardly find more realistic (although still $\mathcal{N}=2$ ) models, in particular ones that have massless hypermultiplets (since this is what would be required to detect the kinetic mixing). A tentative model is given in table 2, which contains the standard-model like group factors. Again the branes must be separated from the orbifold fixed points and orientifold planes. This time, the separations in torus one must be such that $y_{A_{1}}=y_{B_{1}}=y_{C_{1}}$, and $y_{C_{2}}=y_{D}$. This ensures that there is (non-chiral) matter charged under the visible gauge groups, and also some charged (only) under the hidden gauge group $Q_{h}$. Note that stack $D$ is split to $U(1)_{h}$ via two $U(2)$ s in the second and third tori; alternatively it could remain as a stack of two branes and two images, giving a hidden massless $U(1)$ and $S U(2)$. Once more there is kinetic mixing between massless $U(1)$ s; the "hypercharge" is given by

$$
Q_{Y}=\frac{1}{3} Q_{A_{1}}-Q_{A_{2}}+Q_{C_{1}}
$$

Note that, since branes $C_{i}$ are parallel to the orientifold plane, they automatically carry massless gauge groups, and also participate in kinetic mixing. (Because this is still an $\mathcal{N}=2$ model, we will not go on to present the spectrum here.)

The above discussion is of course for a very simple model, and we would of course like to build more realistic examples with $\mathcal{N}=1$ supersymmetry that can then be broken, and genuine chiral matter charged under the correct gauge groups etc. However, if we 


\begin{tabular}{|c||c|c|c||}
\hline stack & $N$ & gauge group & $\left(n^{1}, m^{1}\right) \times\left(n^{2}, m^{2}\right) \times\left(n^{3}, m^{3}\right)$ \\
\hline$A_{1}$ & 6 & $S U(3), Q_{A_{1}}$ & $(1,0) \times(1,1) \times(1,-1)$ \\
$A_{2}$ & 2 & $Q_{A_{2}}$ & $(1,0) \times(1,1) \times(1,-1)$ \\
$B_{1}$ & 4 & $S U(2)$ & $(1,0) \times(0,1) \times(0,-1)$ \\
$C_{1}$ & 2 & $Q_{C_{1}}$ & $(1,0) \times(1,0) \times(1,0)$ \\
\hline$D$ & 4 & $Q_{h}$ & $(1,0) \times(1,-1) \times(1,1)$ \\
$C_{2}$ & 2 & $Q_{C_{2}}$ & $(1,0) \times(1,0) \times(1,0)$ \\
\hline
\end{tabular}

Table 2: Wrapping numbers for a slightly more realistic model.

attempt to realise such models on a torus with an unresolved orbifold we encounter some obstructions. Since we require the cancellation of masses but not of kinetic mixing, we need branes with bulk components that are separated between the hidden and visible $U(1)$ factors in order that they are hidden, and also split into stacks so that they are massless. Thus, given that the mixing in these models is generically large, and that it is difficult (although not impossible) to obtain a truly hidden sector, such models are not of particular interest. More importantly, however, in the current paradigm of LARGE volume [71, 72, 73] or KKLT [27] models we should consider a collection of D6-branes or their T-dual in terms of D3 and D7 branes with gauge fluxes realising the standard model gauge group and spectrum to be a mere local construction supported in some small region of a larger manifold. It is from this perspective that we see that a truly hidden sector separated from the visible one by several string lengths is entirely natural.

Of particular interest are models involving (anti) D3-branes that move in the bulk. These are required for example in the KKLT scenario to uplift to a de Sitter vacuum [27, 28], and may even play the role of an inflaton. Since the charges of the D3-branes can be cancelled by O3-planes (which may be well separated from them), fluxes or D7-branes wrapping cycles with non-trivial curvature, there should be no reason that they may not exhibit kinetic mixing. If the D3-branes and the branes supporting hypercharge are located at generic positions in some Calabi-Yau, the nett effect would be one of volume suppression similar to the flat space case [20]. However in many scenarios, such as KKLT, the hypercharge is placed at a special position, for example the tip of a warped throat. One expects that this could drastically alter the phenomenon of kinetic mixing: not only is the background now warped, but the fluxes that cause the warping also give masses to the very fields that mediate the kinetic mixing. In order to analyse these more general cases, we shall have to go beyond the flat space approximation and develop a supergravity approach.

\section{The supergravity calculation of kinetic-mixing}

As a warm-up exercise for the supergravity approach, let us first demonstrate how one can 
obtain the CFT results of section 2 using only the effective field theory. Masses $m_{a b}$ have been calculated in, for example, Refs. [74, 75], but we shall extend this approach to the computation of kinetic mixing $\chi_{a b}$. To do this, we consider the action of the brane and the supergravity fields $[76,77,78,79]$ :

$$
\begin{aligned}
S_{\mathrm{DBI}} & =\mu_{p} \int d^{p+1} x e^{-\Phi} \sqrt{-\operatorname{det} g+2 \pi \alpha^{\prime} F+B} \\
& \approx \int d^{p+1} x \mu_{p} e^{-\Phi} \sqrt{-g}-\frac{1}{4} \mu_{p} e^{-\Phi} \sqrt{-g}\left(\left(2 \pi \alpha^{\prime}\right)^{2} F_{\mu \nu} F^{\mu \nu}+2\left(2 \pi \alpha^{\prime}\right) F_{\mu \nu} B^{\mu \nu}+B_{\mu \nu} B^{\mu \nu}\right) \\
S_{\mathrm{WZ}} & =\mu_{p} \int_{D p} \sum_{q} C_{q} \wedge \operatorname{tr} \exp \left(2 \pi \alpha^{\prime} F+B\right) \wedge \sqrt{\frac{\hat{A}\left(4 \pi^{2} \alpha^{\prime} R_{T}\right)}{\hat{A}\left(4 \pi^{2} \alpha^{\prime} R_{N}\right)}} \\
S_{\mathrm{R}} & =-\frac{1}{4 \kappa_{10}^{2}} \int d^{10} x(-\operatorname{det} G)^{1 / 2}\left(\left|F_{1}\right|^{2}+\left|\tilde{F}_{3}\right|^{2}+\frac{1}{2}\left|\tilde{F}_{5}\right|^{2}\right) \\
S_{\mathrm{NS}} & =-\frac{1}{4 \kappa_{10}^{2}} \int d^{10} x(-\operatorname{det} G)^{1 / 2} e^{-2 \Phi}\left|H_{3}\right|^{2}
\end{aligned}
$$

where $A_{\mu}$ is a gauge field, $C_{q}$ are the R-R forms and $B_{2}$ is the NS-NS 2 -form and the field-strengths are defined as

$$
\begin{aligned}
F & =d A \\
F_{q+1} & =d C_{q}, \\
H_{3} & =d B_{2}, \\
\tilde{F}_{3} & =F_{3}-C_{0} \wedge H_{3}, \\
\tilde{F}_{5} & =F_{5}-\frac{1}{2} C_{2} \wedge H_{3}+\frac{1}{2} B_{2} \wedge F_{3}, \\
*_{10} \tilde{F}_{5} & =\tilde{F}_{5} .
\end{aligned}
$$

Note that the 2-form $F$ in the first equation above is the usual $F_{\mu \nu}$ and should not be confused with the R-R field strength $F_{2}=d C_{1}$ in the second equation. Also $\mu_{p}=$ $\sqrt{2 \pi}\left(4 \pi^{2} \alpha^{\prime}\right)^{-\frac{1+p}{2}}$ is the brane tension, and $2 \kappa_{10}^{2}=\left(\alpha^{\prime}\right)^{4}(2 \pi)^{7}$.

Already from the Dirac-Born-Infield (DBI) action (4.1) it is clear that the $B$-field can mediate kinetic mixing. In addition, for $\mathrm{D} p$-branes, a $p-1$-form $C_{p-1}$ couples in (4.2) to the gauge fields, which can mediate between branes of the same dimensionality.

\subsection{A simple case without fluxes}

The CFT results for kinetic mixing, e.g., Eq. (2.12) in the D3-D3 system on a toroidal background, are applicable for backgrounds without flux vacuum expectation values. We will now calculate the same results using the supergravity approach based on the DBI action (4.1).

The vertices for the antisymmetric tensor $B_{\mu \nu}$ and $A_{\rho}$ are

$$
\frac{1}{2} 2 \pi \alpha^{\prime} \mu_{p} g_{s}^{-1}\left(k_{\mu} g_{\nu \rho}-k_{\nu} g_{\mu \rho}\right) \delta\left(\Sigma_{p}\right)
$$


for a $p$-brane of worldvolume $\Sigma_{p}$. The propagator for a component of $B_{\mu \nu}, \mu, \nu \in\{0,1,2,3\}$ is straightforward to write down: the diagonal part of the propagator is

$$
G_{\mu \nu ; \rho \sigma}\left(k_{4}, y_{0}, y_{1}\right)=\delta_{\mu \rho} \delta_{\nu \sigma} \frac{2 g_{s}^{2} \kappa_{10}^{2}}{V_{6}} \sum_{k_{6}} \frac{\exp \left[\mathrm{i} k_{6} \cdot\left(y_{1}-y_{0}\right)\right]}{\left|k_{4}\right|^{2}+\left|k_{6}\right|^{2}} .
$$

Here $k_{4}$ and $k_{6}$ are the 4-dimensional and the transverse 6-dimensional momenta (w.r.t $\mu, \nu \in\{0,1,2,3\}$ of $\left.B_{\mu \nu}\right)$, and $y_{1}-y_{0}$ is the 6 -dimensional distance vector in the transverse space.

The $B$-field induced contribution to the 2-point function of gauge fields is

$$
\begin{aligned}
\left\langle A_{\mu_{1}}^{a_{1}} A_{\nu_{1}}^{b_{1}}\right\rangle_{B} & =\frac{\delta}{\delta A_{\mu_{1}}^{a_{1}}} \frac{\delta}{\delta A_{\nu_{1}}^{b_{1}}} \operatorname{tr}_{1} \lambda_{a} \operatorname{tr}_{2} \lambda_{b} \frac{1}{2} \frac{1}{\alpha^{\prime}} \frac{\left(2 \pi \alpha^{\prime}\right)^{3}}{V_{6}}\left(4 \pi^{2} \alpha^{\prime}\right)^{\frac{3-p_{a}}{2}}\left(4 \pi^{2} \alpha^{\prime}\right)^{\frac{3-p_{b}}{2}}\left[A_{\mu}^{a} A_{b}^{\mu} V_{D p_{a}} V_{D p_{b}}\right. \\
& \left.+\left(k_{4}^{2} A_{\mu}^{a} A_{b}^{\mu}-k_{4} \cdot A^{a} k_{4} \cdot A^{b}\right) \int d^{p_{a}-3} y d^{p_{b}-3} y_{1} \sum_{k_{6}} \frac{\exp \left[\mathrm{i} k_{6} \cdot\left(y_{b}-y_{a}\right)\right]}{\left|k_{6}\right|^{2}}\right] .
\end{aligned}
$$

This is the contribution from the $B$-field only. On the torus, there will also be contributions from $C_{p-1}$-forms but only if $p_{a}=p_{b}$. In this case, for rectangular tori, one can show that for brane-brane mixing the $C$-form contribution is equal and opposite to the $B$-contribution, exactly cancelling it; while for brane-antibrane mixing they are equal in sign and magnitude, simply multiplying the above by two. To reproduce the results of Ref. [19], consider braneantibrane mixing on untwisted tori, so that in Neumann-Dirichlet directions the integrals in the above become delta functions; for $p \neq q$ we obtain

$$
\chi_{a b}=g_{a} g_{b} \operatorname{tr}_{1} \lambda_{a} \operatorname{tr}_{2} \lambda_{b} \frac{1}{2 \pi} \frac{l_{s}^{6}}{V_{6}} \frac{\left(V_{a} V_{b}\right)}{l_{s}^{p_{a}+p_{b}-6}} \sum_{n_{i}} \frac{\prod_{i=1}^{N_{\mathrm{DD}}} \exp \left[2 \pi \mathrm{i} \frac{n_{i}}{R_{i}}\left(y_{b}^{i}-y_{a}^{i}\right)\right]}{\sum_{i=1}^{N_{\mathrm{DD}}} n_{i}^{2} l_{s}^{2} / R_{i}^{2}},
$$

where $l_{s}^{2}=2 \pi \alpha^{\prime}$, and $N_{\mathrm{DD}}$ is the number of Dirichlet-Dirichlet directions. For $p=q$ and parallel brane and antibrane the final result is twice the above formula. This agrees with

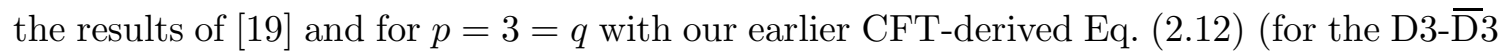
system in the present context of an untwisted toroidal background).

For more general models, where the compact manifold is not a rectangular torus, we consider the action for a single component of $B_{\mu \nu}$ which we shall denote $\phi$, and neglect the transverse modes (the calculation for the $C$-field being identical apart from a minor modification to the vertices):

$$
S=\frac{1}{2 \kappa_{10}^{2}} \int \frac{d^{4} x}{(2 \pi)^{4}} \int_{M^{6}} e^{-2 \Phi}\left(\frac{1}{2} k_{4}^{2} \phi^{2}+\frac{1}{2} d^{(6)} \phi \wedge \star_{6} d^{(6)} \phi\right) .
$$

For a constant dilaton $e^{-2 \Phi}=g_{s}^{-2}$, the Green functions therefore obey

$$
\left(k_{4}^{2}+\Delta_{6}\right) G_{\mu \nu ; \rho \sigma}\left(y_{0}, y_{1}\right)=\delta_{\mu \rho} \delta_{\nu \sigma} 2 \kappa_{10}^{2} g_{s}^{2} \delta\left(y_{1}-y_{0}\right),
$$

where $\Delta_{6}=d \star_{6} d+\left(d d \star_{6}\right)$ is the Laplacian on the compact manifold. To solve the above, we may expand in terms of orthogonal, normalised, eigenfunctions $\phi_{n}$ of the Laplacian (a 
Hermitian operator provided the manifold admits a Hermitian metric) with eigenvalue $\alpha_{n}$, in terms of which the Green function is

$$
G_{\mu \nu ; \rho \sigma}=\delta_{\mu \rho} \delta_{\nu \sigma} 2 \kappa_{10}^{2} g_{s}^{2} \sum_{n} \frac{b_{n}\left(y_{1}\right) \phi_{n}^{*}\left(y_{1}\right)}{\alpha_{n}+k_{4}^{2}} \phi_{n}\left(y_{0}\right),
$$

where $b_{n}\left(y_{1}\right)$ is the weight function. Clearly there is a contribution to the mass term only when $\alpha_{n}=0$, and in all the other contributions, to calculate the mixing we may set $k_{4}^{2}=0$ in the denominator.

Since we are only interested in the fields with indices in the noncompact dimensions (that will couple to the gauge field) the Green function is treated as a zero-form on the compact space. This means that the zero modes of the Laplacian, being harmonic forms, are in one to one correspondence with $H^{0}(M, \mathbb{R})$ : for Calabi-Yau manifolds the only zero mode is the constant solution $\phi_{0}$. This then implies that the result for the mass mixing in Eq. (2.11) applies quite generally and is not particular to the torus.

As a simple example, we apply this to the case of a product of tilted tori, where the metric is $d s^{2}=\sum_{\kappa=1}^{3}(2 \pi)^{2} \frac{T_{2}^{\kappa}}{U_{2}^{\kappa}} d z^{\kappa} d \bar{z}^{\kappa}$ and the periodicities are $z^{\kappa} \sim z^{\kappa}+1, z^{\kappa} \sim z^{\kappa}+U^{\kappa}$. We then have the Laplacian

$$
\Delta_{6}=\partial_{i}\left(\sqrt{g_{6}} g_{6}^{i j} \partial_{j}\right)=\sqrt{g_{6}} \frac{U_{2}^{\kappa}}{(2 \pi)^{2} T_{2}^{\kappa}} \partial_{\kappa} \bar{\partial}_{\kappa}
$$

The eigenfunctions are easily found to be $\frac{1}{\sqrt{V_{6}}} \prod_{\kappa} e^{2 \pi \mathrm{i} p^{\kappa} x^{\kappa}} e^{2 \pi \mathrm{i} q^{\kappa} y^{\kappa}}$, where $z^{\kappa}=x^{\kappa}+U^{\kappa} y^{\kappa}$ and $x^{\kappa} \sim x^{\kappa}+1, y^{\kappa} \sim y^{\kappa}+1$; the weight function is just $\sqrt{g_{6}}$ so we have

$$
G_{\mu \nu ; \rho \sigma}=\delta_{\mu \rho} \delta_{\nu \sigma} \frac{2 \kappa_{10}^{2} g_{s}^{2}}{V_{6}} \sum_{p^{\kappa}, q^{\kappa}} \frac{\prod_{\kappa} e^{\frac{2 \pi \mathrm{i}}{U_{2}} \operatorname{Im}\left(p^{\kappa}\left(y_{0}^{\kappa}-y_{1}\right) \bar{U}^{\kappa}+q^{\kappa}\left(y_{0}^{\kappa}-y_{1}\right)\right)}}{\sum_{\kappa} \frac{1}{T_{2}^{\kappa} U_{2}^{\kappa}}\left|q^{\kappa}+p^{\kappa} \bar{U}^{\kappa}\right|^{2}} .
$$

Using the same vertices as before, we clearly obtain the same result as the string calculation of kinetic mixing in the D3- $\overline{\mathrm{D}} 3$ system found in Eq. (2.12).

\subsection{Inclusion of vacuum expectation values for fluxes}

In type IIB model building it is usually required to include vacuum expectation values (vevs) for the three-form fluxes in order to stabilise the moduli, and so in order to go further, we need to incorporate this into our calculation of kinetic mixing.

Let us begin with a simple observation. The effect of the fluxes in some of the most interesting cases, including KKLT models [27, 28], is that the metric is warped in the vicinity of the standard model branes:

$$
d s^{2}=e^{2 A(y)} \eta_{\mu \nu} d x^{\mu} d x^{\nu}+e^{-2 A(y)} g_{m n} d y^{m} d y^{n} .
$$

If we now restrict our attention to (anti) D3-branes then we can immediately see that, since the coupling of the gauge fields to the antisymmetric tensor and the R-R two-form is classically conformal, the kinetic mixing cannot depend upon the warp factor. Hence all of the modification to the previous cases will derive from the Green function. 
To proceed, we split the fields into $B_{2}=B_{2}^{(4)}+B_{2}^{(6)}+B_{2}^{(46)}$ (and similarly for the twoform $C_{2}$ ) where the superscripts are as follows: (4) indicates both are space-time indices, (6) indicates both are internal, (46) indicates one of each. As we have mentioned, only the components $B_{2}^{(4)}, C_{2}^{(4)}$ can mediate the mixing, but now we wish to give a vev to the components $B_{2}^{(6)}, C_{2}^{(6)}$. There may also be a vev for the five-form field $F_{5}$, but this does not contribute - on the other hand, the contribution of the two-form vevs to $\tilde{F}_{5}$ are crucial.

Importantly, the vevs for $B_{2}^{(6)}, C_{2}^{(6)}$ generate masses for the two-form fields $B_{2}^{(4)}, C_{2}^{(4)}$. In fact, from Eqs. (4.1)-(4.4) we can read off the kinetic and mass terms for $B_{2}$,

$$
\begin{aligned}
S=\frac{1}{2 \kappa_{10}^{2}} \int d^{4} x d^{6} y\left(|\tau|^{2}+\left|C_{2}^{(6)}\right|^{2}\right) \frac{1}{2}\left|H_{3}^{(4)}\right|^{2} & +\frac{1}{8}\left|B_{2}^{(4)}\right|^{2}\left|F_{3}^{(6)}\right|^{2} \\
& +|\tau|^{2}\left|d^{(6)} B_{2}^{(4)}\right|^{2}+\frac{1}{8}\left|C_{2}^{(6)} \wedge d^{(6)} B_{2}^{(4)}\right|^{2},
\end{aligned}
$$

while for $C_{2}$ we have similarly

$$
\begin{aligned}
S=\frac{1}{4 \kappa_{10}^{2}} \int d^{4} x d^{6} y\left(1+\frac{1}{8}\left|B_{2}^{(6)}\right|^{2}\right)\left|F_{3}^{(4)}\right|^{2}+ & \frac{1}{8}\left|C_{2}^{(4)}\right|^{2}\left|H_{3}^{(6)}\right|^{2} \\
& +\left|d^{(6)} C_{2}^{(4)}\right|^{2}+\frac{1}{8}\left|B_{2}^{(6)} \wedge d^{(6)} C_{2}^{(4)}\right|^{2} .
\end{aligned}
$$

Thus fluxes generate masses for the two-form fields; from the string point of view we have stabilised the moduli.

Since the $B_{2}^{(4)}, C_{2}^{(4)}$ fields are now massive this has an effect on the mixing of the $U(1)$ factors which these fields mediate.

Ignoring the non-compact dimensions' kinetic terms, for a component $\phi$ of $C_{\mu \nu}$ we have

$$
\mathcal{L}=\frac{e^{-2 A} \sqrt{g}}{2 \kappa_{10}^{2}}\left[g^{m n} \partial_{m} \phi \partial_{n} \phi+\frac{1}{8}\left|B_{2}^{(6)} \wedge d^{(6)} \phi\right|^{2}+\frac{1}{8}\left|H_{3}^{(6)}\right|^{2} \phi^{2}\right],
$$

where the last term is a mass of $\phi$. We can estimate the magnitude of the effective $\phi$-mass by considering that $H_{3}$ and $F_{3}$ are defined as fluxes threading three-cycles [28],

$$
\begin{aligned}
& \frac{1}{(2 \pi)^{2} \alpha^{\prime}} \int_{A_{K}} H_{3}=m^{K}, \\
& \frac{1}{(2 \pi)^{2} \alpha^{\prime}} \int_{B^{K}} F_{3}=e_{K},
\end{aligned}
$$

where $m^{K}, e_{K}$ are integers and $K=1 . . h^{3}$. Thus we can estimate that

$$
H_{3}, F_{3} \sim n l_{s}^{2} / V_{3}
$$

for some integer $n$ and different three-cycle volumes $V_{3}$. Provided that the cycles threaded by the flux are larger than the string scale, we expect the second term in (4.18) to be less significant, and $\phi$ should behave like a massive scalar with a characteristic length given by $L \sim V_{3} /\left(n l_{s}^{2}\right)$, i.e. the Green functions for the two-form fields behave as

$$
G_{\mu \nu ; \rho \sigma}(y) \propto \delta_{\mu \rho} \delta_{\nu \sigma} e^{-y n l_{s}^{2} / V_{3}} .
$$


The resulting interaction is a "Yukawa type" interaction (whose exponential form derives from the mass of the mediating scalar and has nothing to do with the warping). Thus we expect to be able to probe much of the compact manifold; for $V_{3}$ of $\mathcal{O}(100)$ we can probe $\mathcal{O}(1000)$ string length distances. Note that the three-cycles that are threaded will usually be different for $\mathrm{H}_{3}$ and $F_{3}$. If one of them is much smaller (or the fluxes larger) as is usually the case, then the corresponding two-form is subdominant in the generation of kinetic mixing.

With the full dependence on fluxes to hand, we now proceed to consider specific warped models where we can solve the equations exactly, in order to verify the general behaviour anticipated above. We will consider two cases. The first in the following section is a simplified Randall-Sundrum model which demonstrates that the kinetic mixing is independent of the warping but depends only on the induced mass via the Green function. The second model is the more "realistic" case of kinetic mixing in the Klebanov-Tseytlin throat.

\section{Randall-Sundrum models}

Randall-Sundrum (RS) models [25, 26] involve branes embedded in a slice of $A d S_{5}$. They may be considered as dimensionally reduced string models, or as legitimate phenomenological models in their own right. Since they involve a warped hidden dimension, they are candidates for use as a toy for examining kinetic mixing in a non-trivial background, but still with hope of tractability. Some related work has been performed in [80, 81, 82], under the assumption that the matter fields are not confined to the branes but have wavefunctions extending throughout the fifth dimension. In their case, there were no additional fields, since the wavefunction overlaps contributed to kinetic mixing. We shall rather consider a more string-inspired scenario, where matter fields are confined to branes, and thus shall introduce a string-inspired $B$-field. The metric for the model is taken to be

$$
d s^{2}=e^{-2 k|y|} \eta_{\mu \nu} d x^{\mu} d x^{\nu}+d y^{2},
$$

with $k$ a parameter of the order of the Planck scale.

We shall consider the standard model brane to be a D3-brane at a position $y=0$ in the hidden dimension, and a hidden brane at some position $y_{1}=\pi R$. We shall suppose that there is a massless $U(1)$ field supported upon each, and shall then calculate the mixing. (More generally, we could consider several branes with the $U(1)$ split between them so as to make non-anomalous combinations; the mixing would then be given by calculating the mutual differences.) The Lagrangian of our model is taken to be

$$
\begin{aligned}
\mathcal{L} & =\mathcal{L}_{\text {bulk }}+\mathcal{L}_{\mathrm{D} 3}+\mathcal{L}_{\overline{\mathrm{D}} 3}, \\
\mathcal{L}_{\text {bulk }} & =\frac{M_{5}^{3}}{2 g^{4}} \int \frac{-1}{2} \mathrm{~d} B \wedge *_{5} \mathrm{~d} B+\frac{1}{2} m^{2} B \wedge *_{5} B, \\
\mathcal{L}_{\mathrm{D} 3} & =\frac{1}{4 g^{2}} \int_{\mathrm{D} 3} \frac{1}{2 \pi \alpha^{\prime}} F \wedge *_{4} B+\frac{1}{\left(2 \pi \alpha^{\prime}\right)^{2}} B \wedge *_{4} B, \\
\mathcal{L}_{\overline{\mathrm{D}} 3} & =-\mathcal{L}_{\mathrm{D} 3} .
\end{aligned}
$$


Here we have introduced a $B$-field which will mediate the mixing. The coupling of the $B$-field to the gauge field is specified by the Dirac-Born-Infeld action, but we should point out that it is necessary to introduce three parameters into the model: the coupling of the kinetic term $M_{5}$, the mass-like parameter $m$ and the string mass. If we imagine the above to be derived from a string model, then we expect $M_{5}$ to be related to Planck's constant and the volume of the compactification, and $m$ to be determined by the fluxes; the string scale however generally exists as a free parameter to be determined by experiment. It is tempting to relate the $M_{5}$ coupling to the parameters already extant in the RS scenario; we shall examine the consequences of this later.

To calculate the mixing, as already mentioned we require the Green function, and thus we derive the (very simple) equations of motion

$$
\left[e^{2 k|y|} \eta^{\alpha \beta} \partial_{\alpha} \partial_{\beta}+\partial_{5} \partial_{5}-m^{2}\right] B_{\mu \nu}^{(4)}=0 .
$$

However, from the above action we also find boundary conditions for the $B$-field at the brane:

$$
\partial_{y} B_{\mu \nu}^{(4)}-\left.\frac{M_{s}^{4}}{M_{5}^{3}} B_{\mu \nu}^{(4)}\right|_{y=0, \pi R}=0 .
$$

Since we shall effectively be finding a one-dimensional Green function, these conditions become important; if we were considering a higher dimensional model we could neglect them and consider only the periodic boundary conditions of the compact space.

\subsection{Green functions in one dimension}

The Green functions are straightforward to find for the above action, and the result is a propagator that actually dies more rapidly than the equivalent RS solution at large distances. The procedure for computing them is adapted from Ref. $[81]^{8}$ as follows. Let

$$
\Delta G\left(y, y^{\prime}\right)=\delta\left(y-y^{\prime}\right)
$$

define the Green function (note the loss of translational invariance due to the positions of the branes). Now decompose it into "advanced" and "retarded" components:

$$
G\left(y, y^{\prime}\right)=\theta\left(y-y^{\prime}\right) G_{>}\left(y, y^{\prime}\right)+\theta\left(y^{\prime}-y\right) G_{<}\left(y, y^{\prime}\right),
$$

where $G_{>}, G_{<}$satisfy the homogeneous equation, and we must impose matching conditions at $y=y^{\prime}$. Writing this as

$$
\partial_{y}\left(f(y) \partial_{y} G\left(y, y^{\prime}\right)\right)-h(y) G\left(y, y^{\prime}\right)=k(y) \delta\left(y-y^{\prime}\right)
$$

(with the redundancy deliberate) we obtain the continuity condition $G_{>}(y, y)=G_{<}(y, y)$, and

$$
\partial_{y} G_{>}(y, y)-\partial_{y} G_{<}(y, y)=\frac{k}{2 f}
$$

\footnotetext{
${ }^{8}$ In fact, they derived the propagator for the Randall-Sundrum model at general positions in the bulk at finite momenta. It is possible to extract the information we need from their equation (62) in Ref. [81] by setting the momentum and the parameter $s$ to zero. However, it is actually easier and more transparent to rederive the expression we need.
} 
which sets the normalisation of the propagator. We now solve by separation of variables:

$$
\begin{aligned}
& G_{<}\left(y, y^{\prime}\right) \equiv A_{<}\left(y^{\prime}\right) \tilde{G}_{<}(y), \\
& G_{>}\left(y, y^{\prime}\right) \equiv A_{>}\left(y^{\prime}\right) \tilde{G}_{>}(y),
\end{aligned}
$$

to find the equations

$$
\begin{aligned}
& A_{<}(y) \tilde{G}_{<}^{\prime}(y)-A_{>}(y) \tilde{G}_{>}^{\prime}(y)=\frac{k}{2 f}, \\
& A_{<}(y) \tilde{G}_{<}(y)-A_{>}(y) \tilde{G}_{>}(y)=0 .
\end{aligned}
$$

These allow us to find the $A$ functions. Now, what we desire is $G\left(y_{0}, y_{1}\right)$, where $y_{0}, y_{1}$ are the coordinates of the branes. This is given by $A_{<}\left(y_{1}\right) \tilde{G}_{<}\left(y_{0}\right)$, but these contain values at the boundaries: our boundary conditions are

$$
\begin{aligned}
& \partial \tilde{G}_{<}\left(y_{0}\right)=r \tilde{G}_{<}\left(y_{0}\right), \\
& \partial \tilde{G}_{>}\left(y_{1}\right)=s \tilde{G}_{>}\left(y_{1}\right),
\end{aligned}
$$

and we then find the result

$$
G\left(y_{0}, y_{1}\right)=\frac{k}{2 f}\left(y_{1}\right) \frac{\tilde{G}_{<}\left(y_{0}\right)}{\tilde{G}_{<}^{\prime}\left(y_{1}\right)-s \tilde{G}_{<}\left(y_{1}\right)} .
$$

This is particularly simple to solve numerically; we simply solve one homogeneous equation for $\tilde{G}_{<}(y)$ with initial conditions

$$
\begin{aligned}
& \tilde{G}_{<}\left(y_{0}\right)=C, \\
& \tilde{G}_{<}^{\prime}\left(y_{0}\right)=r C,
\end{aligned}
$$

and the result is independent of the choice of $C$.

For the massive RS action, we can actually solve exactly to obtain

$$
G\left(y_{0}, y_{1}\right)=\frac{4 g^{2}}{M_{5}^{3} m} \frac{1}{\sinh m \pi R} \frac{1}{\left(1-\frac{M_{s}^{8}}{M_{5}^{6} m^{2}}\right)} .
$$

This gives mixing

$$
\chi=g_{a} g_{b} \frac{32 M_{s}^{4}}{M_{5}^{3} m} \frac{1}{\sinh m \pi R} \frac{1}{\left(1-\frac{M_{s}^{8}}{M_{5}^{6} m^{2}}\right)} .
$$

It is tempting to identify $M_{5}$ with $M$ from the existing RS parameters, where $e^{-k \pi R}=$ $M_{\mathrm{SUSY}} / M_{\mathrm{Pl}}$, (so $\left.\pi k R \sim 16 \log 10 \sim 37\right), M_{\mathrm{Pl}}^{2} \approx M^{3} / k$. We also make the assumption that $M_{s}^{8} /\left(M_{5}^{6} m^{2}\right)$ is small. For concreteness, we will take an intermediate string mass of $M_{s}=\sqrt{M_{\mathrm{SUSY}} M_{\mathrm{Pl}}}$. This gives

$$
\begin{aligned}
\chi & \approx g_{a} g_{b} \frac{32}{\log \frac{M_{\mathrm{Pl}}}{M_{\mathrm{SUSY}}}} \frac{M_{s}^{4}}{M_{\mathrm{Pl}}^{2}} \frac{\pi R}{m} \frac{1}{\sinh \pi m R} \\
& \approx g_{a} g_{b} \frac{32}{37} \times \frac{M_{\mathrm{SUSY}}^{2} \pi R}{m} \frac{1}{\sinh \pi m R} .
\end{aligned}
$$


In the limit that $m R \ll 1$, we have

$$
\chi \sim g_{a} g_{b} \times \frac{M_{\mathrm{SUSY}}^{2}}{m^{2}} .
$$

For gauge couplings of order unity, we see that values of $m \sim 10^{4} M_{\text {SUSY }}$ leads to a mixing that is observable in the near future. Comparing this to equation (4.20), if the flux mass is related to a three-cycle in some compact space we should have typical length for a wrapping cycle of $\mathcal{O}\left(10^{2} l_{s}\right)$.

In the opposite limit, $m R \gg 1$, one gets the expected exponential suppression due to the non-zero mass:

$$
\chi \sim g_{a} g_{b} \times \frac{M_{\mathrm{SUSY}}^{2}}{m^{2}}(m \pi R) e^{-m \pi R} .
$$

Thus in RS backgrounds the kinetic mixing can reasonably take any value between zero and the experimental limits, depending on the configuration.

\section{Kinetic mixing on the Klebanov-Tseytlin throat}

We now turn to an example of a Calabi-Yau manifold for which the metric is known, the Klebanov-Tseytlin throat [83, 84]. This is a model of a warped throat region, as found in KKLT models [27]. In this model, there are flux vacuum expectation values, but the back reaction of the flux upon the metric is not fully included. It can thus be seen as an approximation to the Klebanov-Strassler solution [85], where we introduce by hand an "infrared" cutoff $r_{s}$ to model the effect of removing the conical singularity; there is also an "ultraviolet" cutoff $r_{0}$ in both models to render the solution compact (so that $r_{s}<r<r_{0}$ ). In the near-horizon limit it reduces to an RS model, and so we might expect a similar exponential damping effect to occur here. Let us now see if this is the case.

Consider the metric on a general cone:

$$
d s^{2}=h^{-1 / 2}(r) \eta_{\mu \nu} d x^{\mu} d x^{\nu}+h^{1 / 2}(r)\left(d r^{2}+r^{2} d s_{M}^{2}\right)
$$

where $M$ is Sasaki-Einstein maifold. If we put a cutoff at some large radius $r_{0}$ we can consider the above to be part of a larger compact Calabi-Yau manifold (henceforth the bulk). If we wish to use this as a particle physics model, we must then include fluxes to stabilise the various complex structure moduli, which will warp the throat. Moreover, we must consider how to embed the standard model on branes: we may have either D3-branes at the singularity [86] (where we must generalise the above, with for example an orbifold projection) or D3/D7 branes wrapping appropriate cycles elsewhere, either in the throat or the bulk, with $\overline{\mathrm{D}} 3$-branes at the tip $[71,72,73]$ (which can uplift an AdS vacuum to a dS one). Consistency of the model also requires orientifold planes, but they may be present either at the tip of the throat or in the bulk, in the latter case necessitating an image throat. 
The metric on the Klebanov-Tseytlin solution is (conventionally written in the Einstein frame, defined by $d s_{\text {Einstein }}^{2}=\sqrt{g_{s}} d s^{2}$ )

$$
\begin{aligned}
h(r) & =\frac{81\left(g_{s} M \alpha^{\prime}\right)^{2} \log r / r_{s}}{8 r^{4}} \\
& =\frac{27\left(\alpha^{\prime}\right)^{2}\left(2 g_{s} N+3\left(g_{s} M\right)^{2} \log \left(r / r_{0}\right)+3\left(g_{s} M\right)^{2} / 4\right)}{8 r^{4}},
\end{aligned}
$$

where $M$ is the number of fractional D5 branes wrapped on a compact $S^{3} \subset T^{1,1}$ at the tip of the throat, and

$$
d s_{M}^{2}=d s_{T^{1,1}}^{2}=\frac{1}{9}\left(d \psi+\sum_{i=1}^{2} \cos \theta_{i} d \phi_{i}\right)^{2}+\frac{1}{6} \sum_{i=1}^{2}\left(d \theta_{i}^{2}+\sin \theta_{i} d \phi_{i}^{2}\right) .
$$

To calculate the kinetic mixing, we consider the dynamics of the R-R two-form ${ }^{9}, C_{2}^{(4)}$. We start from the action (4.17), and make the assumption that in the throat region, the radial Kaluza-Klein modes are much less significant that the longitudinal modes; this is reasonable since the throat will be long and thin. Again writing $\phi$ for a component of $C_{\mu \nu}$, the action for the relevant term then reduces to

$$
S=\frac{1}{4 g_{s} \kappa_{10}^{2}} \int d^{4} x \partial_{r} \phi \partial_{r} \phi\left(|d r|^{2}+\frac{1}{8}\left|B_{2}^{(6)} \wedge d r\right|^{2}\right)+\frac{1}{8} \phi^{2}\left|H_{3}^{(6)}\right|^{2} .
$$

The vacuum expectation values for the fluxes are taken to be

$$
\begin{aligned}
F_{3} & =\frac{M \alpha^{\prime}}{2} \omega_{3}, \\
H_{3} & =\frac{3 g_{s} M \alpha^{\prime}}{2 r} d r \wedge \omega_{2}, \\
B_{2} & =\frac{3 g_{s} M \alpha^{\prime}}{2} \log \left(r / r_{0}\right) \omega_{2},
\end{aligned}
$$

where

$$
\begin{aligned}
\omega_{3} & =\left(d \psi+\cos \theta_{1} d \phi_{1}+\cos \theta_{2} d \phi_{2}\right) \wedge \omega_{2}, \\
\omega_{2} & =\frac{1}{2}\left(\sin \theta_{1} d \theta_{1} \wedge d \phi_{1}+\sin \theta_{2} d \theta_{2} \wedge d \phi_{2}\right), \\
\omega_{2} \wedge \omega_{3} & =54 \operatorname{Vol}\left(T^{1,1}\right), \\
\omega_{3} & =-\frac{3}{r} *_{6}\left(d r \wedge \omega_{2}\right) .
\end{aligned}
$$

The action then becomes

$$
S=\frac{3 \pi^{3}\left(M \alpha^{\prime}\right)^{2} g_{s}}{4 \kappa_{10}^{2}} \int d^{4} x d r \partial_{r} \phi \partial_{r} \phi\left(2 r \log r / r_{s}+r\left(\log r / r_{0}\right)^{2}\right)+\phi^{2} \frac{1}{r} .
$$

${ }^{9}$ The dynamics of the $B$-field is complicated by its coupling to $C_{2}^{(6)}$, which admits no globally smooth vev due to the non-compact nature of the conifold. If desired, the same analysis can be performed in the large distance limit, giving a similar, but crucially not identical, result. 


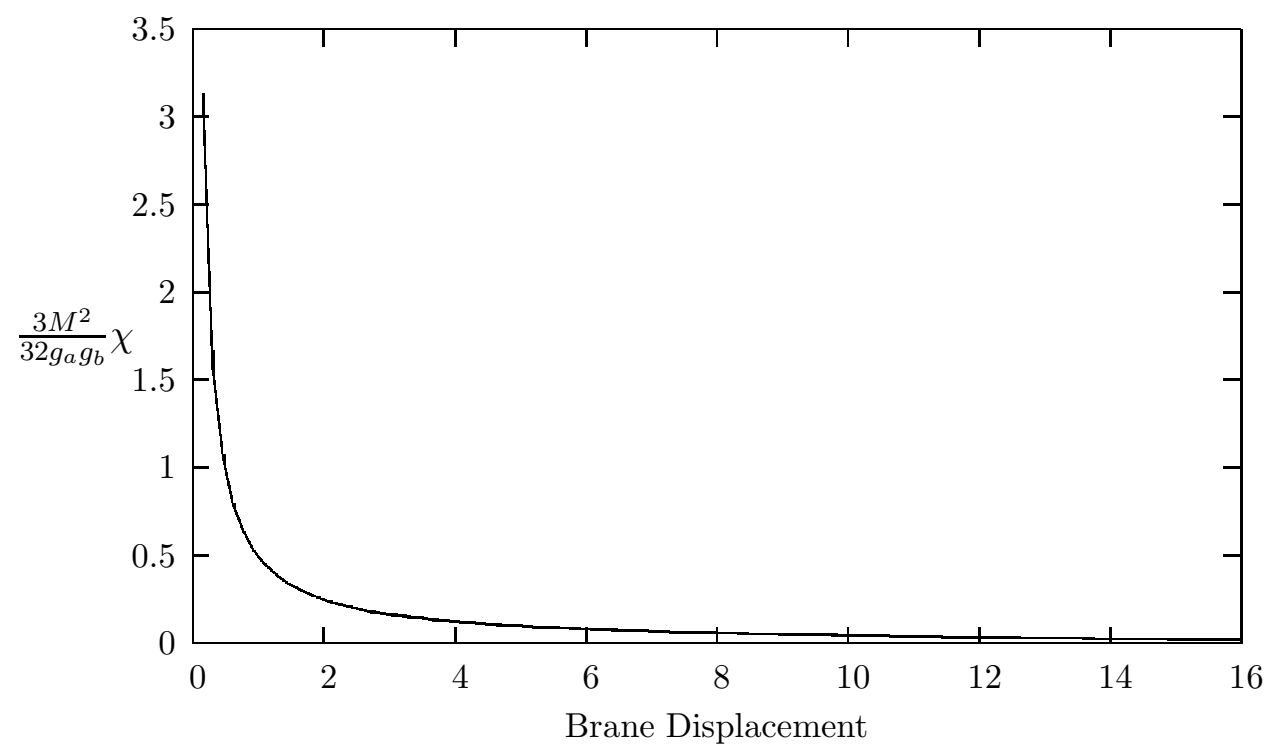

Figure 5: Mixing on the conifold varying with distance of the hidden brane, $y_{1}=\log \left(r_{1} / r_{s}\right)$, up to the mouth of the throat at $y_{1}=16$. ( $M$ is the number of fractional D5 branes wrapped on a compact $S^{3} \subset T^{1,1}$ at the tip of the throat.)

Using the variable $y=\log r / r_{s}$, we then have

$$
S=\frac{3 \pi^{3}\left(M \alpha^{\prime}\right)^{2} g_{s}}{4 \kappa_{10}^{2}} \int d^{4} x d y \partial_{y} \phi \partial_{y} \phi\left(2 y+\left(y-y_{0}\right)^{2}\right)+\phi^{2} .
$$

Note that in the small distance limit, this becomes the equation for a Randall-Sundrum model with a constant mass $\phi$-field, with

$$
\frac{M_{5}^{3}}{g^{2}}=\frac{3 \pi^{3}\left(M \alpha^{\prime}\right)^{2} g_{s}}{\kappa_{10}^{2}} y_{0}^{-2},
$$

and $m^{2}=y_{0}^{-2}$ (note that the dimensions are different). This is quite indicative: in the Klebanov-Tseytlin throat the effective mass is smaller than 1, and moreover for branes in the throat the separation will never exceed the inverse mass - so we do not expect a large suppression.

Using the analysis for the solution of Green functions, we find no contact terms on the brane, and the boundary condition is just that $\partial_{y} \phi=0$ at the branes. We then find

$$
\chi_{a b}=g_{a} g_{b} \frac{32}{3 M^{2}} \frac{1}{\left.4 y_{1}+2\left(y_{1}-y_{0}\right)^{2}\right)} \frac{\tilde{G}_{<}\left(y_{s}\right)}{\tilde{G}_{<}^{\prime}\left(y_{1}\right)},
$$

where the hidden brane is placed at $y_{1}=\log \left(r_{1} / r_{s}\right)$. The homogeneous solutions can be easily found numerically; a graph is given in Fig. 5. Again, the mixing is much larger than might naively have been expected; the Klebanov-Tseytlin throat is comparable to the RS model in the $m R \ll 1$ limit. Once the backreaction of the fluxes becomes important, one might expect an exponential damping of the propagation similar to the $m R \gg 1$ limit 
of the RS model. Therefore in general warped throat configurations it is not possible to place an upper or lower bound on the size of the kinetic mixing between branes: there may indeed be an experimental signal to observe.

\section{Conclusions}

We have shown that models with massless hidden $U(1)$ s can be found in string theory, and argued that they are natural for certain classes of backgrounds. These massless hiddensector $U(1)$ s can nevertheless have observable and experimentally testable effects because they will typically mix with the ordinary photon via a so-called kinetic mixing term. Using conformal field theory and supergravity techniques we have calculated these effects. The latter method can be used even when fluxes are included to stabilise the moduli. Moreover, we have demonstrated that in general kinetic mixing is non-zero even if all the $U(1) \mathrm{s}$ involved are anomaly free and therefore massless. This facilitates extremely sensitive tests in a variety of current and near future low-energy experiments.

The size of the kinetic mixing is model-dependent. Yet, for generic parameter values, it is often within reach of current and near future experiments. There is thus the real possibility that an experimental signal will be observed soon that would give deep insights into the particular string theory background upon which we may live. Alternatively, new stronger bounds will crucially exclude many models. We believe that it is thus worthwhile to examine future models for such fields and the kinetic mixing between them.

\section{Acknowledgements}

MDG would like to sincerely thank the IPPP, Durham for hospitality. AR would like to thank Ralph Blumenhagen, Luis Ibanez, Jan Louis, Dieter Lüst, Fernando Quevedo and Stefan Theisen for enlightening discussions on hidden $U(1)$ s in type II string phenomenology.

\section{A. Remarks on the D3-Dי3 system}

We may wish to follow the procedure outlined in the section 2 for constructing a massless $U(1)$ field from two stacks of branes applied to the D3- $\overline{\mathrm{D}} 3$ system, and start with two stacks: one of two D3-branes, one of two $\overline{\mathrm{D}} 3$-branes. We consider the compact space to be a six-torus, but the discussion regarding the masses applies to any manifold. We then split these into four stacks, giving four gauge fields $A^{\alpha}=\left\{A_{a}^{1}, A_{a}^{2}, A_{\bar{b}}^{1}, A_{\bar{b}}^{2}\right\}$. Due to the mutual supersymmetries preserved, there is only kinetic/mass mixing between the branes and antibranes, not amongst themselves. However, a crucial difference between this system and one of purely branes is that there are uncancelled NS-NS tadpoles, and thus we have a non-zero contribution to the mass from the planar diagrams (with both vertex operators 
on one boundary). The planar and non-panar masses are given by

$$
\begin{aligned}
m_{\text {planar }}^{2} & =\operatorname{tr}\left(\lambda_{a}^{i} \lambda_{a}^{i}\right) \sum_{j} \operatorname{tr}\left(\gamma_{\bar{b}}^{j}\right) m^{2}, \\
m_{\text {non-planar }}^{2} & =\operatorname{tr}\left(\lambda_{a}^{i}\right) \operatorname{tr}\left(\lambda_{\bar{b}}^{j}\right) m^{2},
\end{aligned}
$$

where $m^{2}$ is given by equation (2.11). Thus we can write the Lagrangian as

$$
\mathcal{L} \supset \frac{1}{2}\left(A_{\mu}\right)^{\alpha}(\mathcal{M})_{\alpha \beta}\left(A^{\mu}\right)^{\beta}-\frac{1}{4 g^{2}}\left(F_{\mu \nu}\right)^{\alpha}(\mathcal{X})_{\alpha \beta}\left(F^{\mu \nu}\right)^{\beta},
$$

where

$$
\mathcal{M}=\left(\begin{array}{cccc}
4 m^{2} & 0 & m^{2} & m^{2} \\
0 & 4 m^{2} & m^{2} & m^{2} \\
m^{2} & m^{2} & 4 m^{2} & 0 \\
m^{2} & m^{2} & 0 & 4 m^{2}
\end{array}\right)
$$

and

$$
\mathcal{X}=\left(\begin{array}{cccc}
1 & 0 & -\chi_{11} & -\chi_{12} \\
0 & 1 & -\chi_{21} & -\chi_{22} \\
-\chi_{11} & -\chi_{21} & 1 & 0 \\
-\chi_{12} & -\chi_{22} & 0 & 1
\end{array}\right)
$$

Note that upon diagonalising $\mathcal{M}$, we find no massless $U(1) \mathrm{s}$; all four fields become massive, with masses multiples of $m^{2}$. Note also the presence of the diagonal mass terms from the planar diagrams. This is a new feature present when we have broken supersymmetry, and if we analyse the supergravity calculation we find that it arises from a dilaton tadpole. This occurs because of the uncancelled NS-NS charges present.

\section{References}

[1] F. Quevedo, "Phenomenological aspects of D-branes," Prepared for ICTP Spring School on Superstrings and Related Matters, Trieste, Italy, 18-26 Mar 2002

[2] S. Abel and J. Santiago, "Constraining the string scale: from Planck to Weak and back again," J. Phys. G 30 (2004) R83 [arXiv:hep-ph/0404237].

[3] D. Lüst, "Intersecting brane worlds: A path to the standard model?," Class. Quant. Grav. 21 (2004) S1399 [arXiv:hep-th/0401156].

[4] F. Marchesano, "Progress in D-brane model building," Fortsch. Phys. 55 (2007) 491 [arXiv:hep-th/0702094].

[5] B. Holdom, "Two U(1)'S And Epsilon Charge Shifts," Phys. Lett. B 166 (1986) 196.

[6] R. Foot and X. G. He, "Comment on Z Z-Prime Mixing in Extended Gauge Theories," Phys. Lett. B 267 (1991) 509.

[7] I. Antoniadis, E. Kiritsis and J. Rizos, "Anomalous U(1)s in type I superstring vacua," Nucl. Phys. B 637 (2002) 92 [arXiv:hep-th/0204153].

[8] P. Anastasopoulos, "4D anomalous U(1)'s, their masses and their relation to $6 \mathrm{D}$ anomalies," JHEP 0308 (2003) 005 [arXiv:hep-th/0306042]. 
[9] E. Kiritsis, "D-branes in standard model building, gravity and cosmology," Fortsch. Phys. 52 (2004) 200 [Phys. Rept. 421 (2005 ERRAT,429,121-122.2006) 105] [arXiv:hep-th/0310001].

[10] P. Anastasopoulos, "Anomalous U(1)s masses in non-supersymmetric open string vacua," Phys. Lett. B 588 (2004) 119 [arXiv:hep-th/0402105].

[11] P. Anastasopoulos, M. Bianchi, E. Dudas and E. Kiritsis, "Anomalies, anomalous U(1)'s and generalized Chern-Simons terms," JHEP 0611 (2006) 057 [arXiv:hep-th/0605225].

[12] B. Kors and P. Nath, "A Stueckelberg extension of the standard model," Phys. Lett. B 586 (2004) 366 [arXiv:hep-ph/0402047].

[13] K. Cheung and T. C. Yuan, "Hidden fermion as milli-charged dark matter in Stueckelberg Z' model," JHEP 0703 (2007) 120 [arXiv:hep-ph/0701107].

[14] D. Feldman, Z. Liu and P. Nath, "The Stueckelberg Z' extension with kinetic mixing and milli-charged dark matter from the hidden sector," Phys. Rev. D 75 (2007) 115001 [arXiv:hep-ph/0702123].

[15] B. Holdom, "Oblique electroweak corrections and an extra gauge boson," Phys. Lett. B 259 (1991) 329 .

[16] K. S. Babu, C. F. Kolda and J. March-Russell, "Implications of generalized Z Z' mixing," Phys. Rev. D 57 (1998) 6788 [arXiv:hep-ph/9710441].

[17] W. F. Chang, J. N. Ng and J. M. S. Wu, "A very narrow shadow extra Z-boson at colliders," Phys. Rev. D 74 (2006) 095005 [arXiv:hep-ph/0608068].

[18] J. Kumar and J. D. Wells, "LHC and ILC probes of hidden-sector gauge bosons," Phys. Rev. D 74 (2006) 115017 [arXiv:hep-ph/0606183].

[19] S. A. Abel and B. W. Schofield, "Brane-antibrane kinetic mixing, millicharged particles and SUSY breaking," Nucl. Phys. B 685, 150 (2004) [arXiv:hep-th/0311051].

[20] S. A. Abel, J. Jaeckel, V. V. Khoze and A. Ringwald, "Illuminating the hidden sector of string theory by shining light through a magnetic field," arXiv:hep-ph/0608248.

[21] K. R. Dienes, C. F. Kolda and J. March-Russell, "Kinetic mixing and the supersymmetric gauge hierarchy," Nucl. Phys. B 492 (1997) 104 [arXiv:hep-ph/9610479].

[22] B. Holdom, "Searching for Epsilon Charges and a New U(1)," Phys. Lett. B 178 (1986) 65.

[23] G. Aldazabal, L. E. Ibanez, F. Quevedo and A. M. Uranga, "D-branes at singularities: A bottom-up approach to the string embedding of the standard model," JHEP 0008 (2000) 002 [arXiv:hep-th/0005067].

[24] R. Blumenhagen, M. Cvetic, P. Langacker and G. Shiu, "Toward realistic intersecting D-brane models," Ann. Rev. Nucl. Part. Sci. 55 (2005) 71 [arXiv:hep-th/0502005].

[25] L. Randall and R. Sundrum, "An alternative to compactification," Phys. Rev. Lett. 83 (1999) 4690 [arXiv:hep-th/9906064].

[26] L. Randall and R. Sundrum, "A large mass hierarchy from a small extra dimension," Phys. Rev. Lett. 83 (1999) 3370 [arXiv:hep-ph/9905221].

[27] S. Kachru, R. Kallosh, A. Linde and S. P. Trivedi, "De Sitter vacua in string theory," Phys. Rev. D 68 (2003) 046005 [arXiv:hep-th/0301240]; 
[28] M. Grana, "Flux compactifications in string theory: A comprehensive review," Phys. Rept. 423 (2006) 91 [arXiv:hep-th/0509003].

[29] L. B. Okun, "Limits Of Electrodynamics: Paraphotons?," Sov. Phys. JETP 56 (1982) 502 [Zh. Eksp. Teor. Fiz. 83 (1982) 892].

[30] M. Ahlers, H. Gies, J. Jaeckel, J. Redondo and A. Ringwald, "Laser experiments explore the hidden sector," arXiv:0711.4991 [hep-ph].

[31] J. Jaeckel and A. Ringwald, "A Cavity Experiment to Search for Hidden Sector Photons," Phys. Lett. B 659 (2008) 509 [arXiv:0707.2063 [hep-ph]].

[32] V. V. Popov and O. V. Vasil'ev, "Deviations from Electrodynamics: Sun and Laser," Europhys. Lett. 15 (1991) 7;

[33] J. Redondo, "Helioscope Bounds on Hidden Sector Photons," arXiv:0801.1527 [hep-ph].

[34] E. R. Williams, J. E. Faller and H. A. Hill, "New experimental test of Coulomb's law: A laboratory upper limit on the photon rest mass," Phys. Rev. Lett. 26 (1971) 721.

[35] D. F. Bartlett and S. Loegl, "Limits on an electromagnetic fifth force," Phys. Rev. Lett. 61 (1988) 2285.

[36] H. Gies, J. Jaeckel and A. Ringwald, "Accelerator cavities as a probe of millicharged particles," Europhys. Lett. 76 (2006) 794 [arXiv:hep-ph/0608238].

[37] S. N. Gninenko, N. V. Krasnikov and A. Rubbia, "Search for millicharged particles in reactor neutrino experiments: a probe of the PVLAS anomaly," Phys. Rev. D 75 (2007) 075014 [arXiv:hep-ph/0612203].

[38] M. Glück, S. Rakshit and E. Reya, "The Lamb shift contribution of very light milli-charged fermions," Phys. Rev. D 76 (2007) 091701 [arXiv:hep-ph/0703140].

[39] H. Goldberg and L. J. Hall, "A New Candidate For Dark Matter," Phys. Lett. B 174 (1986) 151.

[40] S. Davidson, B. Campbell and D. C. Bailey, "Limits on particles of small electric charge," Phys. Rev. D 43 (1991) 2314.

[41] A. Melchiorri, A. Polosa and A. Strumia, "New bounds on millicharged particles from cosmology," Phys. Lett. B 650 (2007) 416 [arXiv:hep-ph/0703144].

[42] S. L. Dubovsky, D. S. Gorbunov and G. I. Rubtsov, "Narrowing the window for millicharged particles by CMB anisotropy," JETP Lett. 79 (2004) 1 [Pisma Zh. Eksp. Teor. Fiz. 79 (2004) 3] [arXiv:hep-ph/0311189].

[43] S. Davidson, S. Hannestad and G. Raffelt, "Updated bounds on milli-charged particles," JHEP 0005, 003 (2000) [arXiv:hep-ph/0001179].

[44] S. Davidson and M. E. Peskin, "Astrophysical bounds on millicharged particles in models with a paraphoton," Phys. Rev. D 49 (1994) 2114 [arXiv:hep-ph/9310288].

[45] H. Gies, J. Jaeckel and A. Ringwald, "Polarized light propagating in a magnetic field as a probe of millicharged fermions," Phys. Rev. Lett. 97 (2006) 140402 [arXiv:hep-ph/0607118].

[46] M. Ahlers, H. Gies, J. Jaeckel and A. Ringwald, "On the particle interpretation of the PVLAS data: Neutral versus charged particles," Phys. Rev. D 75 (2007) 035011 [arXiv:hep-ph/0612098]. 
[47] M. Ahlers, H. Gies, J. Jaeckel, J. Redondo and A. Ringwald, "Light from the Hidden Sector," Phys. Rev. D 76 (2007) 115005 [arXiv:0706.2836 [hep-ph]].

[48] R. Cameron et al. [BFRT Collaboration], "Search For Nearly Massless, Weakly Coupled Particles By Optical Techniques," Phys. Rev. D 47 (1993) 3707.

[49] E. Zavattini et al. [PVLAS Collaboration], "Experimental observation of optical rotation generated in vacuum by a magnetic field," Phys. Rev. Lett. 96 (2006) 110406 [arXiv:hep-ex/0507107].

[50] E. Zavattini et al. [PVLAS Collaboration], "New PVLAS results and limits on magnetically induced optical rotation and ellipticity in vacuum," arXiv:0706.3419 [hep-ex].

[51] S. J. Chen, H. H. Mei and W. T. Ni [Q\&A Collaboration], "Q \& A Experiment to Search for Vacuum Dichroism, Pseudoscalar-Photon Interaction and Millicharged Fermions," Mod. Phys. Lett. A 22 (2007) 2815 [arXiv:hep-ex/0611050].

[52] K. Ehret et al. [ALPS Collaboration], "Production and detection of axion-like particles in a HERA dipole magnet: Letter-of-intent for the ALPS experiment," arXiv:hep-ex/0702023; see also http://alps.desy.de

[53] G. Ruoso et al. [BFRT Collaboration], "Limits on light scalar and pseudoscalar particles from a photon regeneration experiment," Z. Phys. C 56 (1992) 505.

[54] C. Robilliard, R. Battesti, M. Fouche, J. Mauchain, A. M. Sautivet, F. Amiranoff and C. Rizzo [BMV Collaboration], "No light shining through a wall," Phys. Rev. Lett. 99 (2007) 190403 [arXiv:0707.1296 [hep-ex]].

[55] A. S. Chou et al. [GammeV (T-969) Collaboration], "Search for axion-like particles using a variable baseline photon regeneration technique," arXiv:0710.3783 [hep-ex].

[56] A. V. Afanasev, O. K. Baker and K. W. McFarlane, "Production and detection of very light spin-zero bosons at optical frequencies," arXiv:hep-ph/0605250.

[57] P. Pugnat et al. [OSQAR Collaboration], CERN-SPSC-2006-035; see http://graybook.cern.ch/programmes/experiments/OSQAR.html

[58] G. Cantatore for the [PLVAS Collaboration], talk at the 3rd Joint ILIAS-CERN-DESY Axion-WIMPs Training Workshop, 19-25 June 2007, University of Patras, Greece; http://axion-wimp.desy.de

[59] E. Masso and J. Redondo, "Compatibility of CAST search with axion-like interpretation of PVLAS results," Phys. Rev. Lett. 97 (2006) 151802 [arXiv:hep-ph/0606163].

[60] J. E. Kim, "PVLAS experiment, star cooling and BBN constraints: Possible interpretation with temperature dependent gauge symmetry breaking," Phys. Rev. D 76 (2007) 051701 [arXiv:0704.3310 [hep-ph]].

[61] I. Antoniadis, A. Boyarsky and O. Ruchayskiy, "Anomaly induced effects in a magnetic field," Nucl. Phys. B 793 (2008) 246 [arXiv:0708.3001 [hep-ph]].

[62] M. Cvetic, G. Shiu and A. M. Uranga, "Three-family supersymmetric standard like models from intersecting brane worlds," Phys. Rev. Lett. 87 (2001) 201801 [arXiv:hep-th/0107143]; M. Cvetic, G. Shiu and A. M. Uranga, "Chiral four-dimensional N = 1 supersymmetric type IIA orientifolds from intersecting D6-branes," Nucl. Phys. B 615 (2001) 3 [arXiv:hep-th/0107166]; 
M. Cvetic, P. Langacker and G. Shiu, "Phenomenology of a three-family standard-like string model," Phys. Rev. D 66 (2002) 066004 [arXiv:hep-ph/0205252];

C. M. Chen, T. Li, V. E. Mayes and D. V. Nanopoulos, "A Realistic World from Intersecting D6-Branes," arXiv:hep-th/0703280.

[63] H. Verlinde, L. T. Wang, M. Wijnholt and I. Yavin, "A Higher Form (of) Mediation," arXiv:0711.3214 [hep-th].

[64] D. Lüst and S. Stieberger, "Gauge threshold corrections in intersecting brane world models," Fortsch. Phys. 55 (2007) 427 [arXiv:hep-th/0302221].

[65] M. Berg, M. Haack and B. Kors, "Loop corrections to volume moduli and inflation in string theory," Phys. Rev. D 71 (2005) 026005 [arXiv:hep-th/0404087].

[66] R. Blumenhagen, S. Moster and T. Weigand, "Heterotic GUT and standard model vacua from simply connected Calabi-Yau manifolds," Nucl. Phys. B 751 (2006) 186. [arXiv:hep-th/0603015].

[67] I. Antoniadis, K. Benakli, A. Delgado, M. Quiros and M. Tuckmantel, "Split extended supersymmetry from intersecting branes," Nucl. Phys. B 744 (2006) 156 [arXiv:hep-th/0601003].

[68] L. J. Dixon, V. Kaplunovsky and J. Louis, "Moduli dependence of string loop corrections to gauge coupling constants," Nucl. Phys. B 355 (1991) 649.

[69] I. Antoniadis, C. Bachas, C. Fabre, H. Partouche and T. R. Taylor, "Aspects of type I - type II - heterotic triality in four dimensions," Nucl. Phys. B 489 (1997) 160 [arXiv:hep-th/9608012].

[70] S. A. Abel and M. D. Goodsell, "Realistic Yukawa couplings through instantons in intersecting brane worlds," JHEP 0710 (2007) 034 [arXiv:hep-th/0612110].

[71] V. Balasubramanian, P. Berglund, J. P. Conlon and F. Quevedo, "Systematics of moduli stabilisation in Calabi-Yau flux compactifications," JHEP 0503 (2005) 007 [arXiv:hep-th/0502058].

[72] J. P. Conlon, F. Quevedo and K. Suruliz, "Large-volume flux compactifications: Moduli spectrum and D3/D7 soft supersymmetry breaking," JHEP 0508 (2005) 007 [arXiv:hep-th/0505076].

[73] J. P. Conlon, S. S. Abdussalam, F. Quevedo and K. Suruliz, "Soft SUSY breaking terms for chiral matter in IIB string compactifications," JHEP 0701 (2007) 032 [arXiv:hep-th/0610129].

[74] D. M. Ghilencea, L. E. Ibanez, N. Irges and F. Quevedo, "TeV-scale Z' bosons from D-branes," JHEP 0208 (2002) 016 [arXiv:hep-ph/0205083].

[75] D. M. Ghilencea, "U(1) masses in intersecting D-brane SM-like models," Nucl. Phys. B 648 (2003) 215 [arXiv:hep-ph/0208205].

[76] Y. K. Cheung and Z. Yin, "Anomalies, branes, and currents," Nucl. Phys. B 517 (1998) 69 [arXiv:hep-th/9710206].

[77] R. Minasian and G. W. Moore, "K-theory and Ramond-Ramond charge," JHEP 9711 (1997) 002 [arXiv:hep-th/9710230].

[78] C. P. Bachas, P. Bain and M. B. Green, "Curvature terms in D-brane actions and their M-theory origin," JHEP 9905 (1999) 011 [arXiv:hep-th/9903210]. 
[79] J. Polchinski, "String theory. Vol. 2: Superstring theory and beyond," Cambridge, UK: Univ. Pr. (1998) $531 p$

[80] T. Gherghetta and A. Pomarol, "Bulk fields and supersymmetry in a slice of AdS," Nucl. Phys. B 586 (2000) 141 [arXiv:hep-ph/0003129].

[81] T. Gherghetta and A. Pomarol, "A warped supersymmetric standard model," Nucl. Phys. B 602 (2001) 3 [arXiv:hep-ph/0012378].

[82] D. Maity, S. Roy and S. SenGupta, "Constraining the Randall-Sundrum modulus in the light of recent PVLAS data," Phys. Rev. D 77, 015010 (2008) [arXiv:0709.3940 [hep-ph]].

[83] I. R. Klebanov and A. A. Tseytlin, "Gravity duals of supersymmetric $\mathrm{SU}(\mathrm{N})$ x $\mathrm{SU}(\mathrm{N}+\mathrm{M})$ gauge theories," Nucl. Phys. B 578 (2000) 123 [arXiv:hep-th/0002159].

[84] C. P. Herzog, I. R. Klebanov and P. Ouyang, "Remarks on the warped deformed conifold," arXiv:hep-th/0108101.

[85] I. R. Klebanov and M. J. Strassler, "Supergravity and a confining gauge theory: Duality cascades and chiSB-resolution of naked singularities," JHEP 0008 (2000) 052 [arXiv:hep-th/0007191].

[86] J. F. G. Cascales, M. P. Garcia del Moral, F. Quevedo and A. M. Uranga, "Realistic D-brane models on warped throats: Fluxes, hierarchies and moduli stabilization," JHEP 0402 (2004) 031 [arXiv:hep-th/0312051]. 